ISSN 1855-3966 (printed edn.), ISSN 1855-3974 (electronic edn.)

\author{
ARS MATHEMATICA CONTEMPORANEA 17 (2019) 153-183 \\ https://doi.org/10.26493/1855-3974.1195.c71 \\ (Also available at http://amc-journal.eu)
}

\title{
Generating polyhedral quadrangulations of the projective plane*
}

\author{
Yusuke Suzuki \\ Department of Mathematics, Niigata University, \\ 8050 Ikarashi 2-no-cho, Nishi-ku, Niigata, 950-2181, Japan
}

Received 9 September 2016, accepted 7 June 2019, published online 17 September 2019

\begin{abstract}
We determine the 26 families of irreducible polyhedral quadrangulations of the projective plane under three reductions called a face-contraction, a 4-cycle removal and a 23 -path shrink, which were first given by Batagelj in 1989. Every polyhedral quadrangulation of the projective plane can be obtained from one of them by a sequence of the inverse operations of the reductions.
\end{abstract}

Keywords: Quadrangulation, projective plane, generating theorem.

Math. Subj. Class.: 05C10

\section{Introduction}

A quadrangulation (resp., triangulation) of a closed surface is a simple graph cellularly embedded on the surface so that each face is quadrilateral (resp., triangular); in particular, a 2-path on the sphere is not a quadrangulation in this paper. It is known that every quadrangulation $G$ of any closed surface is 2 -connected and hence the minimum degree of $G$ is at least 2 . For quadrangulations of closed surfaces, we introduce typical three reductional operations called a face-contraction, a 4-cycle removal and a $22_{3}$-path shrink, which were first given by Batagelj [2]. (See Figure 1. For a formal definition, see the next section.) In this paper, we call the above three operations $\mathcal{P}$-reductions, while call the inverse operations $\mathcal{P}$-expansions.

A quadrangulation of a closed surface is irreducible if no face-contraction is applicable without making a loop or multiple edges. In [20], it was proved that a 4-cycle is the unique irreducible quadrangulation of the sphere, and that there exist precisely two irreducible quadrangulations of the projective plane which are the unique quadrangular embeddings

* This work was supported by JSPS KAKENHI Grant Number 16K05250.

E-mail address: y-suzuki@math.sc.niigata-u.ac.jp (Yusuke Suzuki) 


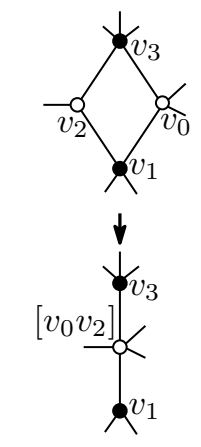

face-contraction

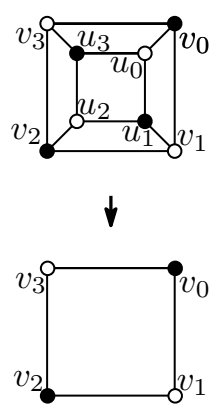

4-cycle removal

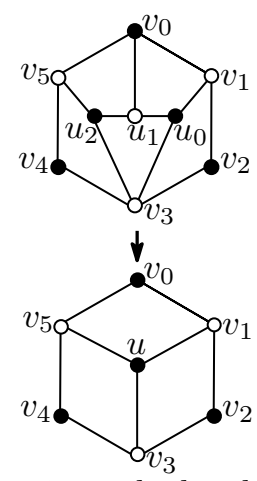

$2_{3}$-path shrink

Figure 1: $\mathcal{P}$-reductions.

of $K_{4}$ and $K_{3,4}$ on the projective plane, respectively (see Figure 2). The irreducible quadrangulations of the torus and the Klein bottle had also been determined in [15] and [14], respectively.
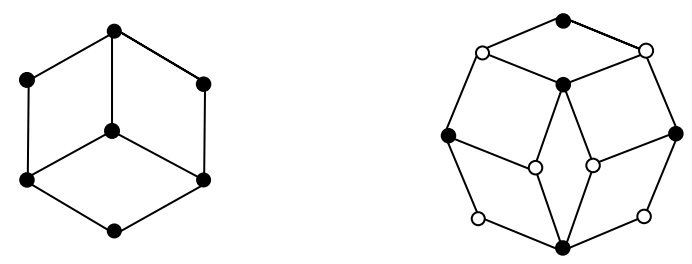

Figure 2: Irreducible quadrangulations of the projective plane where antipodal points of the hexagon and the octagon are identified respectively.

There are some results of quadrangulations of closed surfaces with some conditions. Batagelj [2] proved that any 3-connected quadrangulation on the sphere can be deformed into a cube by a sequence of $\mathcal{P}$-reductions preserving 3 -connectedness. However his proof contained a small mistake, and Brinkmann et al. [3] pointed out it and gave a corrected proof. Observe that a 3 -connected quadrangulation of the sphere corresponds to a 4-regular 3 -connected graph on the same surface by taking its dual. Broersma et al. [4] considered the same problem of the dual version with weaker conditions than Brinkmann et al. [3]. Nakamoto [17] discussed quadrangulations with minimum degree 3 and proved that any quadrangulation of the sphere (resp., the projective plane) with minimum degree 3 can be deformed into a pseudo double wheel (resp., a Möbius wheel or the unique quadrangular embedding of $K_{3,4}$ on the projective plane) by a sequence of face-contractions and 4-cycle removals, preserving the minimum degree at least 3. Brinkmann et al. [3] also proved the same result only on the sphere using a restricted face-contraction. Furthermore, the results in [13] implies that every 3 -connected quadrangulation of a closed surface $F^{2}$ except the sphere can be reduced into one of irreducible quadrangulations of $F^{2}$ by $\mathcal{P}$-reductions, preserving the 3 -connectedness. In addition, the recent study [25] discussed another reductional operation defined for 3-connected quadrangulations of closed surfaces. 
Let $G$ be a graph embedded on a non-spherical closed surface $F^{2}$. The representativity of $G$, denoted by $r(G)$, is the minimum number of intersecting points of $G$ and $\gamma$, where $\gamma$ ranges over all essential simple closed curves on the surface. A graph $G$ embedded on $F^{2}$ is $r$-representative if $r(G) \geq r$ (see [22] for the details). A graph $G$ embedded on a closed surface $F^{2}$ is polyhedral if $G$ is 3-connected and 3-representative. For example, each of two quadrangulations in Figure 2 is 3 -connected but not polyhedral since these embeddings have representativity 2. Observe that all facial walks in a polyhedral embedded graph $G$ are cycles, and any two of them are either disjoint, intersect in one vertex, or intersect in one edge. From such a point of view, polyhedral embedded graphs are frequently regarded as "good" embeddings in topological graph theory (see e.g., [8, 9, 10, 11]); note that every simple triangulation of a closed surface is polyhedral, while simple quadrangulations are not necessarily so. Furthermore, it is known that there is one to one correspondence between the set of polyhedral quadrangulations of a nonspherical closed surface $F^{2}$ (resp., 3 -connected quadrangulations of the sphere) and the set of optimal 1-embeddings of $F^{2}$ (resp., optimal 1-planar graphs of the sphere, see [5, 6, 12, 21, 23, 24] for definitions and some results).

A face $f=v_{0} v_{1} v_{2} v_{3}$ of a polyhedral quadrangulation $G$ of $F^{2}$ is $\mathcal{P}$-contractible (or simply contractible) if a face-contraction at either $\left\{v_{0}, v_{2}\right\}$ or $\left\{v_{1}, v_{3}\right\}$ results in another polyhedral quadrangulation of the same surface. Similarly, we define "P -removable (or simply removable)" and "P-shrinkable (or simply shrinkable)" for a 4-cycle $C$ and a 2path $P$, both of which are induced by vertices of degree 3, respectively. A polyhedral quadrangulation $G$ of $F^{2}$ is $\mathcal{P}$-irreducible if $G$ has none of a contractible face, a removable 4 -cycle and a shrinkable 2-path. The following is our main theorem in this paper. In the figures, to obtain the projective plane, identify antipodal pairs of points of each hexagon or octagon.

Theorem 1.1. There are precisely 26 families of $\mathcal{P}$-irreducible quadrangulations of the projective plane presented in Figures 8,11 and 16. Every polyhedral quadrangulation of the projective plane can be obtained from one of them by a sequence of $\mathcal{P}$-expansions.

This paper is organized as follows. In the next section, we define basic terminology and reductional operations for quadrangulations. In Section 3, we show some lemmas to prove Theorem 1.1. In Section 4, we determine inner structures of 2-cell regions bounded by 4 , 5 or 6 -cycles of $\mathcal{P}$-irreducible quadrangulations. Furthermore in Section 5 , we consider ones bounded by several 6 or 8 -walks. Before proving the main theorem, we classify $\mathcal{P}$ irreducible quadrangulations with attached cubes into five types in Section 6. The last section is devoted to prove Theorem 1.1.

\section{Basic definitions}

We denote the vertex set and the edge set of a graph $G$ by $V(G)$ and $E(G)$, respectively. A $k$-path (resp., $k$-cycle) in a graph $G$ means a path (resp., cycle) of length $k$. (We define the length of a path (or cycle) by the number of its edges.) We say that $S \subset V(G)$ is a cut of a connected graph $G$ if $G-S$ is disconnected. In particular, $S$ is called a $k$-cut if $S$ is a cut with $|S|=k$. A cycle $C$ of $G$ is separating if $V(C)$ is a cut.

Let $G$ be a graph 2-cell embedded on a closed surface $F^{2}$. That is, each connected component of $F^{2}-G$ is homeomorphic to an open 2-cell (or an open disc), which is called a face of $G$. We denote the face set of $G$ by $F(G)$. A facial cycle $C$ of a face $f$ is a cycle bounding $f$ in $G$; i.e., $C=\partial f$. Furthermore in our argument, we often discuss the interior 
of a 2-cell region $D$ bounded by a closed walk $W$ of $G$, i.e., $W=\partial D$, which contains some vertices and edges. (Note that a 2 -cell region implies an "open" 2 -cell region in this paper.) Then, $\bar{D}$ (resp., $\bar{f}$ ) denotes a closure of $D$ (resp., $f$ ), i.e., $\bar{D}=D \cup \partial D$ (resp., $\bar{f}=f \cup \partial f$ ). Let $f_{1}, \ldots, f_{k}$ denote the faces of $G$ incident to $v \in V(G)$ where $\operatorname{deg}(v)=k$. Then, the boundary walk of $\bar{f}_{1} \cup \cdots \cup \bar{f}_{k}$ is the link walk of $v$ and denoted by $l w(v)$. Clearly, $l w(v)$ bounds a 2-cell region containing a unique vertex $v$.

A simple closed curve $\gamma$ on a closed surface $F^{2}$ is trivial if $\gamma$ bounds a 2-cell on $F^{2}$, and $\gamma$ is essential otherwise. Furthermore, $\gamma$ is surface separating if $F^{2}-\gamma$ is disconnected. Clearly, a trivial closed curve on $F^{2}$ is always separating, whereas an essential one is either separating or not. We apply these definitions to cycles of graphs embedded in the surface, regarding them as simple closed curves. It is an important property of the projective plane that any two essential simple closed curves are homotopic to each other.

Let $G$ be a quadrangulation of a closed surface $F^{2}$ and let $f$ be a face of $G$ bounded by a cycle $v_{0} v_{1} v_{2} v_{3}$. (For brevity, we also use the notation like $f=v_{0} v_{1} v_{2} v_{3}$.) The face-contraction of $f$ at $\left\{v_{0}, v_{2}\right\}$ in $G$ is to identify $v_{0}$ and $v_{2}$, and replace the two pairs of multiple edges $\left\{v_{0} v_{1}, v_{2} v_{1}\right\}$ and $\left\{v_{0} v_{3}, v_{2} v_{3}\right\}$ with two single edges respectively. In the resulting graph, let $\left[v_{0} v_{2}\right]$ denote the vertex arisen by the identification of $v_{0}$ and $v_{2}$. See the left-hand side of Figure 1. The inverse operation of a face-contraction is called a vertex-splitting. If the graph obtained from $G$ by a face-contraction is not simple, then we do not apply it.

Let $G$ be a quadrangulation of a closed surface $F^{2}$, and let $f$ be a face of $G$ bounded by $v_{0} v_{1} v_{2} v_{3}$. A 4 -cycle addition to $f$ is to put a 4-cycle $C=u_{0} u_{1} u_{2} u_{3}$ inside $f$ in $G$ and join $v_{i}$ and $u_{i}$ for each $i \in\{0,1,2,3\}$. The inverse operation of a 4-cycle addition is called a 4-cycle removal (of $C$ ), as shown in the center of Figure 1. We call the subgraph $H$ isomorphic to a cube with eight vertices $u_{i}, v_{i}$ for $i \in\{0,1,2,3\}$ an attached cube. We denote $\partial(H)=v_{0} v_{1} v_{2} v_{3}$, and we call $C$ an attached 4-cycle of $H$.

As mentioned in the introduction, there exist some results of 3-connected quadrangulations (or quadrangulations with minimum degree 3 ) of closed surfaces; see [2, 3, 13, 17] for example. In those results, the 4-cycle removal is necessary by the following reason: Let $\tilde{G}$ denote the resulting graph obtained from a 3 -connected quadrangulation $G$ of a closed surface by applying 4 -cycle additions to all faces of $G$. Clearly $\tilde{G}$ is 3 -connected, however we cannot apply any face-contraction to $\tilde{G}$ without making a vertex of degree 2 .

In [3, 17], pseudo double wheels $W_{2 k}(k \geq 3)$ and a Möbius wheels $\tilde{W}_{2 k-1}(k \geq 2)$ are treated as minimal quadrangulations of the sphere and the projective plane, respectively; for their formal definitions, see [17]. However, the following third reduction can reduce a pseudo double wheel $W_{2 k}(k \geq 4)$ into $W_{2(k-1)}$. That is, $W_{2 k}$ can be deformed into a cube by $k-3$ such reductions.

Assume that a polyhedral quadrangulation $G$ of a closed surface $F^{2}$ has a vertex $u$ of degree 3. (Every 3-connected quadrangulation of either the sphere or the projective plane has such a vertex of degree 3 , by Euler's formula.) Let $v_{0} v_{1} \cdots v_{5}$ be a 6 -cycle bounding a 2-cell region $D$ on $F^{2}$, which contains a unique vertex $u$ and we assume that $v_{1}, v_{3}$ and $v_{5}$ are neighbors of $u$. The $2_{3}$-vertex splitting of $u$ is the expansion of $G$, defined as follows:

(i) Delete $u$ and the three edges incident to $u$.

(ii) Put a 2-path $u_{0} u_{1} u_{2}$ into the interior of $D$ and add edges $u_{0} v_{1}, u_{0} v_{3}, u_{1} v_{0}, u_{2} v_{3}$ and $u_{2} v_{5}$. 
Note that each of $u_{0}, u_{1}$ and $u_{2}$ has degree 3 in the resulting graph. The inverse operation of a $23_{3}$-vertex splitting is called a $2_{3}$-path shrink, as shown in the right-hand side of Figure 1.

Similarly to the case of 4 -cycle removals, it is not difficult to see that $2_{3}$-path shrinks are necessary, when considering $\mathcal{P}$-irreducible quadrangulations; replace an attached cube with a graph having a long path consisting of vertices of degree 3 under some conditions. Now, we have defined all the operations in the paper. Note that all of them preserve the bipartiteness of quadrangulations of closed surfaces.

\section{Lemmas}

To prove our main theorem, we show some lemmas which state properties of polyhedral $(\mathcal{P}$-irreducible) quadrangulations of closed surfaces. We first give the following proposition which is however clear by the definition of polyhedral quadrangulations.

Proposition 3.1. A polyhedral quadrangulation has no vertex of degree 2.

The following holds not only for quadrangulations but also for even embeddings of closed surfaces $F^{2}$, that is, a graph on $F^{2}$ with each face bounded by a cycle of even length. Taking a dual of an even embedding and using the odd point theorem, it is easy to show the following.

Lemma 3.2. An even embedding of a closed surface has no separating closed walk of odd length.

The length of two cycles in an even embedding of a closed surface $F^{2}$ have the same parity if they are homotopic to each other on $F^{2}$ (see [1, 7, 16]). Furthermore, it is wellknown that any two essential closed curves on the projective plane are homotopic to each other, and hence the following holds.

Lemma 3.3. The length of two essential cycles in an even embedding of the projective plane have the same parity.

When classifying $\mathcal{P}$-irreducible quadrangulations in the latter half of the paper, we focus on whether such a quadrangulation is bipartite or non-bipartite.

Lemma 3.4. If a quadrangulation $G$ of the projective plane admits an essential cycle of even (resp., odd) length, then $G$ is bipartite (resp., non-bipartite).

Proof. If $G$ admits an essential cycle of even length, then every essential cycle of $G$ has even length by the previous lemma. Of course, all trivial cycles of $G$ is separating and hence have even length by Lemma 3.2. Therefore, $G$ is bipartite.

We denote the set of vertices of a graph $G$ with degree $i$ by $V_{i}(G)$ (or simply $V_{i}$ ). In this paper, we often focus on the subgraph of $G$ induced by $V_{3}$, and denote it by $\left\langle V_{3}\right\rangle_{G}$. In [17], the following lemma was proved.

Lemma 3.5. Let $G$ be a quadrangulation of a closed surface $F^{2}$ with minimum degree at least 3 and assume that $\left\langle V_{3}\right\rangle_{G}$ contains a cycle $C$ of length $k$. Then $k \geq 3$ and one of the followings holds;

(i) if $k=4$, then $G$ is a cube on the sphere or $C$ is an attached 4-cycle of an attached cube in $G$, 
(ii) if $k$ is odd, then $G$ is a Möbius wheel $\tilde{W}_{k}$ on the projective plane,

(iii) if $k$ is even and at least 6 , then $G$ is a pseudo double wheel $W_{k}$ on the sphere.

Let $G$ be a quadrangulation of a closed surface $F^{2}$ and let $f=v_{0} v_{1} v_{2} v_{3}$ be a face of $G$. Then a pair $\left\{v_{i}, v_{i+2}\right\}$ is called a diagonal pair of $f$ in $G$ for each $i \in\{0,1\}$. A closed curve $\gamma$ on $F^{2}$ is a diagonal $k$-curve for $G$ if $\gamma$ passes only through distinct $k$ faces $f_{0}, \ldots, f_{k-1}$ and distinct $k$ vertices $x_{0}, \ldots, x_{k-1}$ of $G$ such that for each $i, f_{i}$ and $f_{i+1}$ share $x_{i}$, and that for each $i,\left\{x_{i-1}, x_{i}\right\}$ forms a diagonal pair of $f_{i}$ of $G$, where the subscripts are taken modulo $k$. Furthermore, we call a simple closed curve $\gamma$ on $F^{2}$ a semidiagonal $k$-curve if in the above definition $\left\{x_{i-1}, x_{i}\right\}$ is not a diagonal pair for exactly one $i$; note that $x_{i-1} x_{i}$ is an edge of $\partial f_{i}$ in this case.

Lemma 3.6. Let $G$ be a quadrangulation of a closed surface $F^{2}$ with a 2 -cut $\{x, y\}$. Then there exists a surface separating diagonal 2-curve for $G$ only through $x$ and $y$.

Proof. Observe that every quadrangulation of any closed surface $F^{2}$ is 2-connected and admits no such closed curve on $F^{2}$ crossing $G$ at most once. Thus there exists a surface separating simple closed curve $\gamma$ on $F^{2}$ crossing only $x$ and $y$, since $\{x, y\}$ is a cut of $G$.

We shall show that $\gamma$ is a diagonal 2-curve. Suppose that $\gamma$ passes through two faces $f_{1}$ and $f_{2}$ meeting at two vertices $x$ and $y$. If $\gamma$ is not a diagonal 2-curve, then $x$ and $y$ are adjacent on $\partial f_{1}$ or $\partial f_{2}$. Since $G$ has no multiple edges between $x$ and $y$, and since $\{x, y\}$ is a 2-cut of $G$, we may suppose that $x$ and $y$ are adjacent in $\partial f_{1}$, but not in $\partial f_{2}$. Here we can take a separating 3 -cycle of $G$ along $\gamma$. This contradicts Lemma 3.2.

Lemma 3.7. Let $G$ be a 3-connected quadrangulation of a closed surface $F^{2}$, and let $f=v_{0} v_{1} v_{2} v_{3}$ be a face of $G$. If the face-contraction of $f$ at $\left\{v_{0}, v_{2}\right\}$ violates the 3connectedness of the graph but preserves the simplicity, then $G$ has a separating diagonal 3 -curve passing through $v_{0}, v_{2}$ and another vertex $x \in V(G)-\left\{v_{0}, v_{1}, v_{2}, v_{3}\right\}$.

Proof. Let $G^{\prime}$ be the quadrangulation of $F^{2}$ obtained from $G$ by the face-contraction of $f$ at $\left\{v_{0}, v_{2}\right\}$. Since $G^{\prime}$ has connectivity $2, G^{\prime}$ has a 2-cut. By Lemma 3.6, $G^{\prime}$ has a separating diagonal 2-curve $\gamma^{\prime}$ passing through two vertices of the 2-cut. Clearly, one of the two vertices must be $\left[v_{0} v_{2}\right]$ of $G^{\prime}$, which is the image of $v_{0}$ and $v_{2}$ by the contraction of $f$; otherwise, $G$ would not be 3 -connected, a contradiction. Let $x$ be another vertex of $G^{\prime}$ on $\gamma^{\prime}$ other than $\left[v_{0} v_{2}\right]$. Note that $x$ is not a neighbor of $\left[v_{0} v_{2}\right]$ in $G^{\prime}$.

Now apply the vertex-splitting of $\left[v_{0} v_{2}\right]$ to $G^{\prime}$ to recover $G$. Then a diagonal 3-curve for $G$ passing through only $v_{0}, v_{2}$ and $x$ arises from $\gamma^{\prime}$ for $G^{\prime}$.

Lemma 3.8. Let $G$ be a 3-representative quadrangulation of a non-spherical closed surface $F^{2}$ and let $f=v_{0} v_{1} v_{2} v_{3}$ be a face of $G$. If the face-contraction of $f$ at $\left\{v_{0}, v_{2}\right\}$ yields another quadrangulation with representativity at most 2 but preserves the simplicity, then $G$ has either an essential diagonal 3-curve or an essential semi-diagonal 3-curve, which passes through $v_{0}, v_{2}$ and another vertex $x \in V(G)-\left\{v_{0}, v_{1}, v_{2}, v_{3}\right\}$.

Proof. Let $G^{\prime}$ be the quadrangulation of the non-spherical closed surface $F^{2}$ obtained from $G$ by a face-contraction of $f$ at $\left\{v_{0}, v_{2}\right\}$. If the representativity of $G^{\prime}$ is at most 1 , then $G$ would have an essential simple closed curve crossing with $G$ at most twice, contrary to $G$ being 3-representative. Thus $G^{\prime}$ has representativity 2 and hence $G^{\prime}$ admits either an essential diagonal 2-curve or an essential semi-diagonal 2-curve. Similarly to Lemma 3.7, 
one of the two vertices passed by the curve must be $\left[v_{0} v_{2}\right]$ of $G^{\prime}$ and $G$ has an essential diagonal (resp., semi-diagonal) 3-curve when the former (resp., the latter) case happens.

The following lemmas show properties of $\mathcal{P}$-irreducible quadrangulations of non-spherical closed surfaces. To simplify our statements, we suppose that $G$ represents a $\mathcal{P}$ irreducible quadrangulation of a non-spherical closed surface $F^{2}$ hereafter in this section.

Lemma 3.9. If $G$ has a 4-cycle $C=v_{0} v_{1} v_{2} v_{3}$ bounding a 2-cell region $D$, then there is no face $f$ of $G$ in $D$ such that one of the diagonal pairs of $f$ is $\left\{v_{0}, v_{2}\right\}$ or $\left\{v_{1}, v_{3}\right\}$.

Proof. Suppose, for a contradiction, that $G$ has a 4-cycle $C=v_{0} v_{1} v_{2} v_{3}$ bounding a 2-cell region $D$ and a face $f$ bounded by $a v_{1} c v_{3}$ in $D$. We assume that $D$ contains as few vertices of $G$ as possible. We denote the subgraph of $G$ in $\bar{D}$ by $H$; note that $H$ can be regarded as a quadrangulation of the sphere.

Since $C$ is separating, we have $\partial f \neq C$. Furthermore, $G$ is $\mathcal{P}$-irreducible and hence $f$ is not $\mathcal{P}$-contractible at $\{a, c\}$. If the face-contraction at $\{a, c\}$ breaks the simplicity of the graph, then $G$ has edges $\{a x, c x\}$ for $x \in V(G)-\left\{v_{1}, v_{3}\right\}$. (Clearly, it does not have a loop.) If $x \in V(G)-V(H)$, we would have $\partial f=C$, contrary to our assumption. Thus, we may assume that $x$ is either $v_{0}$ or $v_{2}$, now say $v_{0}$; observe that $v_{0} \neq a, c$ in this case. Now $G$ would have an edge $a v_{0}$ (or $c v_{0}$ ) and it contradicts Lemma 3.2.

By the above argument, the face-contraction at $\{a, c\}$ does not break the simplicity, hence it breaks the 3 -connectedness or the property of representativity at least 3 . That is, we find either a surface separating diagonal 3-curve or an essential diagonal 3-curve (or an essential semi-diagonal 3-curve) passing through $f$ and $\{a, c\}$ by Lemmas 3.7 and 3.8. In each case, if $\{a, c\} \cap\left\{v_{0}, v_{2}\right\}=\emptyset$, then $f$ could not be passed by such a diagonal curve. Therefore we may suppose that $a=v_{0}$ and $c \neq v_{2}$.

By Lemma 3.2 again, there is not an edge joining $c$ and $v_{2}$. Thus, we can find a face $f^{\prime}$ of $H$ one of whose diagonal pairs is $\left\{c, v_{2}\right\}$. Let $C^{\prime}$ be the 4 -cycle $v_{1} v_{2} v_{3} c$ of $G$. Since $\operatorname{deg}(c) \geq 3$, we have $\partial f^{\prime} \neq C^{\prime}$. Therefore, $C^{\prime}$ and $f^{\prime}$ are a 4 -cycle and a face which satisfy the assumption of the lemma, and moreover, $C^{\prime}$ can cut a strictly smaller graph than $H$ from $G$. Thus, this contradicts the choice of $C$.

Lemma 3.10. Let $f=v_{0} v_{1} v_{2} v_{3}$ be a face of $G$. If the face-contraction of $f$ at $\left\{v_{0}, v_{2}\right\}$ breaks the simplicity of the graph, then there is a vertex $x \in V(G)-\left\{v_{1}, v_{3}\right\}$ adjacent to both of $v_{0}$ and $v_{2}$ such that $v_{0} v_{1} v_{2} x$ is an essential 4-cycle in $G$. In particular, if $F^{2}$ is the projective plane, then $G$ is bipartite.

Proof. First, assume that the face-contraction yields a loop. Then, we have $v_{0} v_{2} \in E(G)$. By Lemma 3.2, $v_{0} v_{1} v_{2}$ should be an essential 3-cycle. However, we would find an essential simple closed curve intersecting $G$ at only $v_{0}$ and $v_{2}$, contrary to $G$ being 3-representative.

Therefore, we may assume that the face-contraction yields multiple edges. Under the conditions, there should be a vertex $x \in V(G)-\left\{v_{0}, v_{1}, v_{2}, v_{3}\right\}$ which is adjacent to both of $v_{0}$ and $v_{2}$. If a 4-cycle $v_{0} v_{1} v_{2} x$ is trivial and bounds a 2-cell region $D$, then $D$ and $f$ would satisfy the conditions of Lemma 3.9, a contradiction. Therefore $v_{0} v_{1} v_{2} x$ should be essential. If $F^{2}$ is the projective plane, then $G$ is bipartite by Lemma 3.4.

Lemma 3.11. If $G$ has a trivial diagonal 3-curve $\gamma$, then the disc bounded by $\gamma$ contains the unique vertex, which has degree 3 . 
Proof. Suppose that $\gamma$ passes through three vertices $\left\{v_{0}, v_{1}, v_{2}\right\}$ and three faces $\left\{f_{0}, f_{1}, f_{2}\right\}$ where each $f_{i}$ is bounded by $v_{i} a_{i} v_{i+1} b_{i}$ so that the 6 -cycle $v_{0} b_{0} v_{1} b_{1} v_{2} b_{2}$ bounds a 2 -cell region $D$ including $f_{i}$ and $a_{i}$ for $i \in\{0,1,2\}\left(v_{3}=v_{0}\right)$. Suppose, for a contradiction, that $D$ contains at least two vertices. That is, this implies that $a_{0}, a_{1}$ and $a_{2}$ could not be identified to one vertex. Thus, we can find a vertex $a_{i} \neq a_{i+1}, a_{i+2}$, now say $a_{0}\left(a_{0} \neq a_{1}, a_{2}\right)$.

If there is an edge joining $a_{0}$ and $v_{2}$, then we can find a 2-cell region $D^{\prime}$ bounded by $a_{0} v_{2} b_{2} v_{0}$. Since $a_{0} \neq a_{2}, D^{\prime}$ is not a face of $G$. Furthermore we have $\operatorname{deg}\left(a_{2}\right) \geq 3$ and hence the region bounded by $a_{0} v_{2} a_{2} v_{0}$ is not a face of $G$ and includes at least one vertex. This means that $D^{\prime}$ satisfies the conditions of Lemma 3.9, a contradiction. Therefore, we conclude that $a_{0} v_{2} \notin E(G)$.

Now consider the face-contraction of $f_{0}$ at $\left\{a_{0}, b_{0}\right\}$. Since $G$ is $\mathcal{P}$-irreducible, $G$ should have a diagonal 3 -curve or a semi-diagonal 3-curve passing through three vertices $\left\{a_{0}, b_{0}, x\right\}$ for $x \in V(G)-\left\{a_{0}, b_{0}\right\}$. (Note that the face-contraction clearly preserves the simplicity of the graph by the above argument, i.e., $a_{0} v_{2} \notin E(G)$.) Since $a_{0}$ is an inner vertex of $D, x$ must be a vertex of $\partial D$.

However, since $a_{0} \neq a_{1}, a_{2}, x$ must coincide with $v_{2}$. Since $a_{0} v_{2} \notin E(G)$ again, there should be a face whose diagonal pair is $\left\{a_{0}, v_{2}\right\}$, but it contradicts Lemma 3.2. Hence, we can conclude that $D$ contains exactly one vertex $a_{0}\left(=a_{1}=a_{2}\right)$ and the lemma follows.

Lemma 3.12. Let $f=v_{0} v_{1} v_{2} v_{3}$ be a face of $G$ with $\operatorname{deg}\left(v_{0}\right), \operatorname{deg}\left(v_{2}\right) \geq 4$.

(i) If $F^{2}$ is the projective plane, then a face-contraction of $f$ at $\left\{v_{1}, v_{3}\right\}$ preserves the 3-connectedness.

(ii) If $F^{2}$ is not the projective plane and if a face-contraction of $f$ at $\left\{v_{1}, v_{3}\right\}$ breaks the 3-connectedness, then $G$ has an essential separating diagonal 3-curve $\gamma$ passing through $v_{1}, v_{3}$ and another vertex $x \in V(G)-\left\{v_{0}, v_{1}, v_{2}, v_{3}\right\}$.

Proof. The statement (ii) immediately follows from Lemmas 3.7 and 3.11. In the projectiveplanar case, we cannot take such an essential separating diagonal 3-curve $\gamma$.

Lemma 3.13. The induced subgraph $\left\langle V_{3}\right\rangle_{G}$ has no vertex of degree 3.

Proof. Suppose, for a contradiction, that $G$ has a vertex $v$ with $\operatorname{deg}(v)=3$ and each of its three neighbors also has degree 3 (see the left-hand side of Figure 3). Note that the boundary of the hexagon is a cycle of $G$; otherwise, it would disturb the simplicity of $G$, Lemma 3.2, Lemma 3.9 or the property of representativity at least 3 . We can easily find a trivial separating diagonal 3 -curve passing through $\left\{v_{0}, v_{1}, v_{2}\right\}$ and that the 3 -cut cuts off the four vertices, contrary to Lemma 3.11.

Suppose that the induced subgraph $\left\langle V_{3}\right\rangle_{G}$ of a $\mathcal{P}$-irreducible quadrangulation $G$ has a path $P=u_{0} u_{1} u_{2}$ of length 2 . Then the configuration around $P$ becomes the center of Figure 3. The following lemma refers to the non-shrinkability of $P$.

Lemma 3.14. Let $P=u_{0} u_{1} u_{2}$ be a 2-path in $G$ induced by vertices of degree 3 (as shown in the center of Figure 3) and assume that $\operatorname{deg}\left(v_{4}\right) \geq 4$. Then, there is an essential diagonal 3 -curve or an essential semi-diagonal 3-curve passing through $\left\{v_{0}, u_{1}, v_{2}\right\}$. 

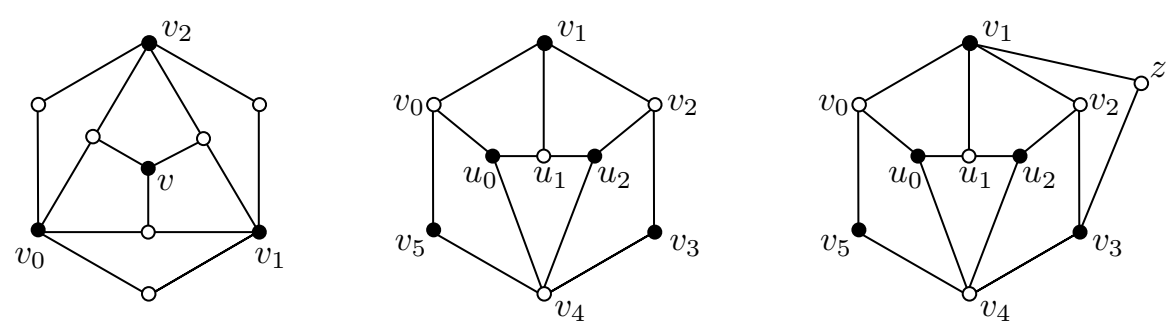

Figure 3: Partial structures of $\mathcal{P}$-irreducible quadrangulations.

Proof. Apply the $23_{3}$-path shrink to $P$ and denote $G^{\prime}$ be the resulting graph. Let $u$ be a vertex of $G^{\prime}$ which is the shrunk image of $P$; note that $u$ is adjacent to $v_{0}, v_{2}$ and $v_{4}$. Since $G$ is $\mathcal{P}$-irreducible, $G^{\prime}$ is not a polyhedral quadrangulation. If $G^{\prime}$ is not simple, $u v_{0}$ and $u v_{2}$ must be multiple edges. This implies that $v_{0}=v_{2}$, however this also implies that $G$ is not simple or $v_{1}$ has degree 2 in $G$, a contradiction.

Next, we assume that $G^{\prime}$ has a 2-cut. By Lemma 3.6, $G^{\prime}$ has a separating diagonal 2 -curve $\gamma^{\prime}$ passing through $\left\{v_{0}, v_{2}\right\}$; otherwise, $G$ would have a 2 -cut. Now we can find a separating diagonal 3-curve $\gamma$ in $G$ corresponding to $\gamma^{\prime}$ naturally. Note that $\gamma$ is not a semi-diagonal 3-curve by Lemma 3.2. Let $f=v_{0} x v_{2} y$ be the third face passed by $\gamma$, which lies outside of the hexagon bounded by $v_{0} v_{1} v_{2} v_{3} v_{4} v_{5}$. If $\gamma$ is essential, then we are done. Therefore, we assume that $\gamma$ is trivial. If neither of $x$ and $y$ corresponds to $v_{1}$, then we have got a contradiction by Lemma 3.9. Thus, one of $x$ and $y$, say $x$, corresponds to $v_{1}$. This means that $\operatorname{deg}\left(v_{1}\right)=3$, however, it contradicts Lemma 3.13.

Finally, assume that $G^{\prime}$ has representativity at most 2. Similarly, $G^{\prime}$ has an essential diagonal 2-curve or an essential semi-diagonal 2-curve passing through $\left\{v_{0}, v_{2}\right\}$. We can easily find our required essential curve passing through $\left\{v_{0}, u_{1}, v_{2}\right\}$ of $G$.

Lemma 3.15. The induced subgraph $\left\langle V_{3}\right\rangle_{G}$ has no path of length at least 3 .

Proof. Suppose to the contrary that $G$ has such a path $P=u_{0} u_{1} u_{2} v_{2}$ (see the right-hand side of Figure 3). By the above lemma, $z$ should coincide with $v_{0}$. However, $v_{1} z$ would become multiple edges, a contradiction.

Lemma 3.16. Assume that $G$ has an attached cube $H$ with $\partial(H)=v_{0} v_{1} v_{2} v_{3}$, an attached 4-cycle $C=u_{0} u_{1} u_{2} u_{3}$ and $u_{i} v_{i} \in E(G)$ for each $i \in\{0,1,2,3\}$. Then there is an essential diagonal (or semi-diagonal) 3-curve $\gamma$ passing through $\left\{v_{0}, u_{1}, v_{2}\right\}$ or $\left\{v_{1}, u_{2}, v_{3}\right\}$.

Proof. Apply the 4-cycle removal of $C$ to $G$ and let $G^{\prime}$ denote the resulting graph. It is clear that the 4-cycle removal clearly preserves the simplicity of the graph. Thus, first suppose that $G^{\prime}$ is not 3-connected. By Lemma 3.6, we can find a separating diagonal 2-curve $\gamma^{\prime}$ in $G^{\prime}$ passing through $\left\{v_{0}, v_{2}\right\}$ or $\left\{v_{1}, v_{3}\right\}$. If $\gamma^{\prime}$ is trivial, then it contradicts Lemma 3.9. If $\gamma^{\prime}$ is essential, we can find our requied diagonal 3-curve $\gamma$ in $G$.

Therefore, we may assume that $G^{\prime}$ has representativity at most 2 and has an essential diagonal (or semi-diagonal) $k$-curve $\gamma^{\prime}$ where $k$ is at most 2. If $\gamma^{\prime}$ does not pass through a face $f=v_{0} v_{1} v_{2} v_{3}$, then $G$ also has representativity at most 2, contrary to our assumption. Thus, $\gamma^{\prime}$ passes through $f$ and two vertices $\left\{v_{0}, v_{2}\right\}$ or $\left\{v_{1}, v_{3}\right\}$ and we got our conclusion. (Note that $\gamma^{\prime}$ does not pass through two neighboring vertices of $v_{0} v_{1} v_{2} v_{3}$. Otherwise, $\gamma^{\prime}$ would be an essential semi-diagonal 2-curve also in $G$.) 
For an attached cube $H$ with $\partial(H)=v_{0} v_{1} v_{2} v_{3}$, we call a pair of two vertices $\left\{v_{i}, v_{i+2}\right\}$ a cube diagonal pair of $H$ for each $i \in\{0,1\}$. In particular, a cube diagonal pair is facing if they are on a boundary cycle of a face $f$ of $G$ outside the 2-cell region bounded by $\partial(H)$. According to the above argument, an essential diagonal (or semi-diagonal) 3-curve passes through $f$.

\section{Regions bounded by 4-, 6- or 8-cycles}

Consider a disk $D$ bounded by a cycle $C=v_{0} v_{1} \cdots v_{2 m-1}$ of length $2 m$. Put a vertex $x$ into the center of $D$ and join it to $v_{2 i}$ for each $i \in\{0, \ldots, m-1\}$. Then, the resulting disk quadrangulation is a pseudo wheel and denoted by $W_{2 m}^{-}$.

Lemma 4.1. Let $G$ be a quadrangulation of a closed surface $F^{2}$ and let $D$ be a 2-cell region bounded by a closed walk $C$ of length 4,6 or 8 such that

(i) there is at least one vertex inside $D$,

(ii) all vertices inside D have degree at least 3 and

(iii) D does not have a unique vertex $x$ of degree 4 such that $l w(x)=C$ (when $|C|=8$ ).

Then, there exists a vertex of degree 3 inside $D$.

Proof. Let $H$ be a graph contained in $\bar{D}$. It suffices to prove the case when $C$ is a cycle. (Even if $C$ is not a cycle, i.e., there exists a vertex appearing twice on $C$, the analogous proof works.) We use induction on $|V(H)|$. Let $v_{0}, \ldots, v_{m-1}$ be vertices lying on $C$ in this order for some $m \in\{4,6,8\}$. The initial step of the induction is the case that $|V(H)|=7$. In this case, $H$ must be isomorphic to $W_{6}^{-}$and its center vertex has degree 3 . (When the length of $C$ equals 4 , it is not difficult to list up all the (disc) quadrangulations with at most 7 vertices, e.g., see [19]. Every such graph has a vertex of degree 2 not lying on any specified outer cycle.) Thus, we suppose that $|V(H)| \geq 8$ in the following argument.

First, assume that there is a diagonal of $C$. Since at least one of the two regions separated by the diagonal satisfies Conditions (i) - (iii), there is a vertex of degree 3 inside the region by the induction hypothesis. Thus, we suppose that there is no diagonal in $D$.

Furthermore, suppose that there is a vertex $x$ joining two vertices $v_{i}$ and $v_{i+2}$. Then, the 2-path $v_{i} x v_{i+2}$ separates $D$ into a quadrilateral region $D^{\prime}$ and the other region $D^{\prime \prime}$. If $D^{\prime}$ contains a vertex, then the induction hypothesis works immediately. Thus, we may assume that $D^{\prime}$ contains no vertex. Further, if $D^{\prime \prime}$ contains at least one vertex and $G \cap \overline{D^{\prime \prime}}$ is not isomorphic to $W_{8}^{-}$, then we can also apply the induction hypothesis. When the case that $G \cap \overline{D^{\prime \prime}}$ is isomorphic to $W_{8}^{-}$, the unique inner vertex $y$ of $D^{\prime \prime}$ should be adjacent to $x$, and hence $x$ has degree 3 ; otherwise, the degree of $x$ would become 2 .

Therefore, we suppose that $D^{\prime \prime}$ contains no vertex. Under the condition, there should be edges joining $x$ and alternate vertices on $C$ so that $H$ becomes disc quadrangulation since $C$ has no diagonal. Then, $H$ is isomorphic to $W_{8}^{-}$since $|V(H)| \geq 8$. However, it contradicts (iii).

By the above arguments, we may assume that $D$ contains no diagonal and no 2-path joining $v_{i}$ and $v_{i+2}$. This implies that all vertices $v_{i}$ of $C$ have degree at least 3 . When $|C|$ is equal to 6 or 8 , add an extra vertex $\hat{x}$ outside $D$ and join it to alternate vertices to obtain a quadrangulation $\hat{H}$ of the sphere; if $|C|=4$, then we do nothing and let $\hat{H}=H$. Observe that $\hat{H}$ has minimum degree at least 3 . 
By Euler's formula, we have $\left|V_{3}(\hat{H})\right| \geq 8$. Even if $|C|=8$, the number of vertices of degree 3 on $C$ is at most 4 by our construction of $\hat{H}$. Therefore, the lemma follows.

The following lemma is important to determine the inner structures of 2-cell regions of $\mathcal{P}$-irreducible quadrangulations bounded by closed walks of length 4,6 or 8 .

Lemma 4.2. Let $G$ be a $\mathcal{P}$-irreducible quadrangulation of a non-spherical closed surface and let $D$ be a 2-cell region bounded by a closed walk $W=w_{0} w_{1} \cdots w_{k-1}$ for some $k \in\{4,6,8\}$. Suppose that $W$ does not bound a face of $G$ and that $G \cap \bar{D}$ is not isomorphic to an attached cube. Then $G \cap \bar{D}$ includes;

(i) a diagonal edge (when $k \in\{6,8\}$ ),

(ii) a 2-path $w_{i} x w_{i+2}$,

(iii) a 2-path $w_{i} x w_{i+4}$ (when $k=8$ and $w_{i} \neq w_{i+4}$ ),

(iv) a 3-path (or a 3 -cycle if $w_{i}=w_{i+3}$ ) $w_{i} x y w_{i+3}$ (when $k \in\{6,8\}$ ) or

(v) a 4-cycle $w_{i} x y z w_{i+4}$ (when $k=8$ and $w_{i}=w_{i+4}$ ),

where $x, y$ and $z$ are distinct inner vertices of $D$ and the indices are taken modulo $k$.

Proof. In this proof, we call a path (or a cycle) in the statement a short path of $D$. Suppose, for a contradiction, that $D$ includes no short path. By Lemma 4.1, $D$ contains a vertex of degree 3 as an inner vertex; since if $D$ has a unique vertex, then it clearly includes a short path of type (ii). First, assume that $D$ contains a vertex $u_{i}$ of degree 3 of an attached cube $Q$; where $Q$ consists of a 4 -cycle $C=u_{0} u_{1} u_{2} u_{3}$ induced by vertices of degree 3 and $\partial(Q)=v_{0} v_{1} v_{2} v_{3}$ with an edge $u_{i} v_{i}$ for each $i \in\{0,1,2,3\}$. We consider the cases depending on the order of $V(C) \cap V(W)$.

Case I. $|V(C) \cap V(W)|=1$ (assume $w_{0}=u_{0}$ ): Then $u_{0}$ would have a vertex of degree at least 4 , contrary to the assumption.

Case II. $|V(C) \cap V(W)|=2$ : If such vertices are diagonal vertices of $C$, say $u_{0}$ and $u_{2}$, then we have $\operatorname{deg}\left(u_{0}\right) \geq 4$, as well as the above case. Thus, we suppose that such two vertices are adjacent on both of $C$ and $W$, say $w_{0}=u_{0}$ and $w_{1}=u_{1}$. Note that $u_{2}$ and $u_{3}$ are inner vertices in this case. Since $\operatorname{deg}\left(w_{0}\right)=\operatorname{deg}\left(w_{1}\right)=3, v_{0}$ (resp., $\left.v_{1}\right)$ should coincide with $w_{k-1}$ (resp., $w_{2}$ ). In this case, $v_{2}$ and $v_{3}$ are inner vertices of $D$; otherwise $D$ would contain a short path (ii) or (iii). However, $w_{2} v_{2} v_{3} w_{k-1}$ would become (iv) if $k \in\{6,8\}$; note that if $k=4$, then $w_{k-1} w_{2}$ would form multiple edges since $\operatorname{deg}\left(w_{0}\right)=\operatorname{deg}\left(w_{1}\right)=3$.

Case III. $|V(C) \cap V(W)|=3$ : We can easily exclude this case, since the unique inner vertex of $C$ is adjacent to two vertices of $W$ and it would form either (ii) or (iii).

Case IV. $V(C) \cap V(W)=\emptyset$ : By Lemma 3.16, at least one of cube diagonal pairs, say $\left\{v_{0}, v_{2}\right\}$, should be facing. We further divide this case into the following subcases.

Case IV-a. $W$ is a cycle of $G$ : Then both of $v_{0}$ and $v_{2}$ should be vertices of $W$. Note that by Lemma 3.2, $\left\{v_{0}, v_{2}\right\}$ coincides with $\left\{w_{i}, w_{i+2}\right\}$ or $\left\{w_{i}, w_{i+4}\right\}$. If one of $v_{1}$ and $v_{3}$ is an inner vertex of $D$, then $D$ clearly would contain a 2-path of (ii) or (iii) in the lemma. Therefore, they also should be vertices of $W$. However, if $k$ equals 6 or 8 , then $D$ would 
have a diagonal edge (i), on the other hand, if $k=4$, then it corresponds to an attached cube, contrary to our assumption.

Case IV-b. $W$ is not a cycle: Note that we only have to consider the case of $k \in\{6,8\}$. This case is further divided into the following subcases.

Case IV-b-1. $w_{i}=w_{i+3}$ (say $\left.w_{0}=w_{3}\right)$ : Note that $G$ is nonbipartite since it includes an essential cycle of odd length. Now we may suppose that an essential simple closed curve of Lemma 3.16 passes through such a vertex $w_{0}=w_{3}$. We may suppose that $v_{0}=w_{0}$ in this case and there should be the edge $v_{2} w_{3}$ (see (a) in Figure 4). In the figure, we find a hexagonal region bounded by $W^{\prime}=w_{0} w_{1} w_{2} w_{3} v_{2} v_{1}$. If there is no identification of vertices of $W^{\prime}$, then we would have a short path $w_{0} v_{1} v_{2} w_{3}$ of type (iv). Even if there is such an identification, we find either a short path (i) or (ii), a contradiction.

Case IV-b-2. $w_{i}=w_{i+4}$ (assume $w_{0}=w_{4}$ ): Similarly to the above arguments, we assume that $v_{0}=w_{0}$ and there is a face bounded by $v_{2} s w_{4} t$ in $G$ where $s, t \in V(G)$. If there is no identification of vertices of closed walk $W^{\prime \prime}=w_{0} w_{1} w_{2} w_{3} w_{4} s v_{2} v_{1}$ bounding an octagonal region, there would be a short path of type (v). When there is identification of vertices of $W^{\prime \prime}$, we pay attention to the simplicity and the representativity of the whole graph; e.g., if $v_{1}=s$, we would have multiple edges $w_{0} v_{1}$. In any case, we find our required short path.

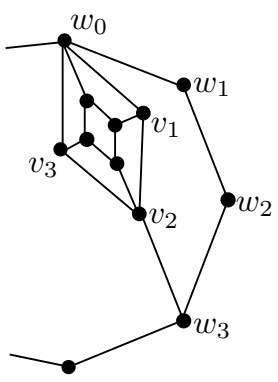

(a)

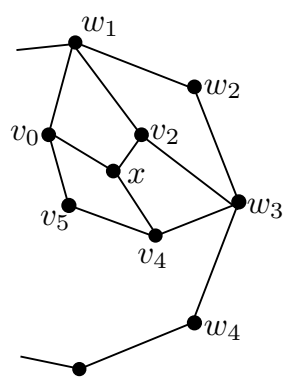

(b)

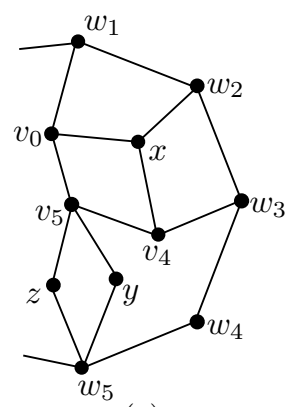

(c)

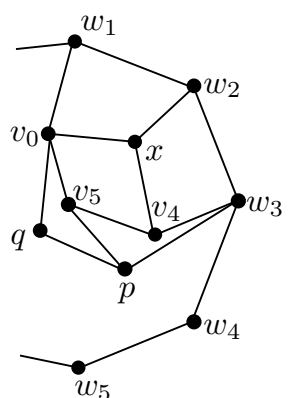

(d)

Figure 4: Inside of a region bounded by closed walks of length 4, 6 or 8 .

Therefore after this, we may assume that $D$ does not contain a vertex of a 4 -cycle induced by vertices of degree 3 , that is, each inner vertex of degree 3 is on the path of $\left\langle V_{3}\right\rangle_{G}$ with length at most 2, by Lemmas 3.5, 3.13 and 3.15; note that a Möbius wheel in Lemma 3.5 is not polyhedral. We can take an inner vertex $x$ of degree 3 so as to be an endpoint of a path of $\left\langle V_{3}\right\rangle_{G}$; otherwise, each path of $\left\langle V_{3}\right\rangle_{G}$ would join two vertices of $W$, contrary to our assumption and Lemmas 3.2 and 3.15.

Let $l w(x)=v_{0} v_{1} v_{2} v_{3} v_{4} v_{5}$ be the link walk of $x$ and assume that $v_{0}, v_{2}$ and $v_{4}$ are adjacent to $x$ and that $\operatorname{deg}\left(v_{0}\right), \operatorname{deg}\left(v_{2}\right) \geq 4$. Now we apply the face-contraction of $x v_{0} v_{1} v_{2}$ at $\left\{x, v_{1}\right\}$, and denote the resulting graph by $G^{\prime}$.

We first assume that $G^{\prime}$ is not simple. By Lemma 3.10, there is an edge joining $v_{1}$ and $v_{4}$ in $G$ such that a cycle $v_{1} v_{2} x v_{4}$ of $G$ is essential. Suppose that the edge $v_{1} v_{4}$ is in $D$. Clearly, $W$ is not a cycle, and we may assume that $k=8$ and that $w_{0}=w_{4}=v_{4}$. However, it easily follows that there exists a short path passing through $x$. Also in the case that $v_{1} v_{4}$ runs outside of $D, v_{1}$ and $v_{4}$ should be vertices of $W$ and hence we can find a 
short path. Then, assume that $G^{\prime}$ is simple in the following argument.

Next, we assume that either the representativity or connectivity of $G^{\prime}$ is at most 2 . In each case, $G$ has an essential diagonal (or semi-diagonal) 3-curve $\gamma$ passing through $x$ and $v_{1}$ by Lemmas 3.8 and 3.12. In fact, there are some cases depending on the positions and identifications of vertices in $D$. However, in each case, the similar argument holds and hence we prove only one substantial case below, for the sake of brevity.

Here, we consider the case that $\gamma$ passes through $\left\{v_{1}, x, v_{3}\right\}$ and that $v_{1}=w_{1}$ and $v_{3}=w_{3}$ (see (b) in Figure 4). In this case, if $v_{2} \neq w_{2}, D$ would contain a 2-path $w_{1} v_{2} w_{3}$ of type (ii) in the lemma. Therefore we suppose $v_{2}=w_{2}$. Note that each of $v_{0}, v_{4}$ and $v_{5}$ is an inner vertex of $D$ and that there is no edge $v_{l} w$ for $l \in\{0,4,5\}$ and $w \in V(W)$; otherwise, there would be a short path.

Next, we assume $\operatorname{deg}\left(v_{4}\right) \geq 4$ and consider the face-contraction of $v_{0} x v_{4} v_{5}$ at $\left\{v_{5}, x\right\}$. By the above argument, $v_{5}$ has no adjacent vertex of $W$ and hence we do not have to care about the simplicity of the resulting graph. Thus, similarly to the above argument, we can find a face $v_{5} y w_{5} z$ in $D$ by Lemmas 3.8 and 3.12, where either $w_{1}=w_{5}$ or $w_{2}=w_{5}$, i.e., $W$ is not a cycle of $G$. We assume $w_{1}=w_{5}$ here. (The case when $w_{2}=w_{5}$ can be shown in a similar way.) See (c) in Figure 4. Actually, $k \neq 4$ in this case. Note that $y$ and $z$ are inner vertices of $D$ and further note that $\{y, z\} \cap\left\{v_{0}, v_{4}\right\}=\emptyset$ by the above argument. It also implies that $\operatorname{deg}\left(v_{5}\right) \geq 4$ and $\operatorname{deg}\left(w_{5}\right) \geq 4$.

By Lemmas 3.8 and 3.12 again and by Lemma 3.2, there should be diagonal 3-curve $\gamma^{\prime \prime}$ passing $z, y$ and $w \in V(W)$; note that semi-diagonal 3-curve is not suitable since each of $y$ and $z$ is not adjacent to a vertex of $V(W)$. In this case, we have $k=8$ and $w=w_{0}=w_{4}$ since if $w=w_{4}=w_{6}, w_{4} w_{5}$ and $w_{5} w_{6}$ become multiple edges. However in this case, we find a short path (iv) of length 3 linking $w_{0}$ and $w_{5}$ (or a short path (iii) of length 2 linking $w_{5}$ and $\left.w_{7}\right)$.

Therefore, suppose that $\operatorname{deg}\left(v_{4}\right)=3$ and there is a face $w_{3} v_{4} v_{5} p$ where $p$ is an inner vertex of $D$; otherwise we would find a short path. Observe that $\operatorname{deg}\left(w_{3}\right) \geq 4$ in this case. Furthermore, if $\operatorname{deg}\left(v_{5}\right) \geq 4$, then we consider the face-contraction of $w_{3} v_{4} v_{5} p$ at $\left\{p, v_{4}\right\}$. Similarly to the above argument, there must be a face $p s w_{6} t$ where $w_{2}=w_{6}$ since $x$ and $v_{0}$ are inner vertices of $D$ and hence there is an essential diagonal 3-curve passing through $\left\{w_{2}, v_{4}, p\right\}$. However, we find a short 3-path $w_{3} p s w_{6}$ in this case.

Hence, we may assume that $\operatorname{deg}\left(v_{5}\right)=3$ and there is a face $v_{0} v_{5} p q$ (see (d) in Figure 4). Then there is a 2-path $x v_{4} v_{5}$ induced by vertices of degree 3. By Lemma 3.14, there should be an essential diagonal (or semi-diagonal) 3 -curve passing through $\left\{w_{2}, v_{4}, p\right\}$. Similarly, we can find a short path around it. (For example, if $w_{2}=w_{5}$ and the edge $p w_{5} \in E(G)$ exists, then we find a short path $w_{3} p w_{5}$ of type (ii).) Thus, the lemma follows.

Figure 5 shows some partial structures of polyhedral quadrangulations of closed surfaces, each of which is bounded by a trivial 4 -cycle $v_{0} v_{1} v_{2} v_{3}$. The center graph in the figure has a 4 -cycle $u_{0} u_{1} u_{2} u_{3}$ induced by vertices of degree 3 and hence this partial structure is an attached cube. Recall that if a polyhedral quadrangulation is $\mathcal{P}$-irreducible and has an attached cube, then one of two cube diagonal pairs is facing by Lemma 3.16. Next, see the right-hand side of Figure 5. For a natural number $n, Q_{2}^{(n)}$ represents the graph having the following structure: There are $n+1$ internally vertex-disjoint paths of length 2 between $v_{0}$ and $v_{2}$, including $v_{0} v_{1} v_{2}$ and $v_{0} v_{3} v_{2}$, so that they divide the region bounded by $v_{0} v_{1} v_{2} v_{3}$ into $n$ quadrilateral regions each of which has the structure $Q_{2}$ having a facing cube diagonal pair $\left\{v_{0}, v_{2}\right\}$. Note that $Q_{2}^{(1)}$ corresponds to $Q_{2}$. 


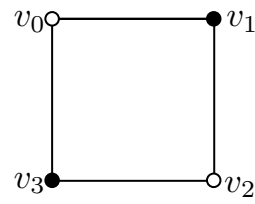

$Q_{1}$

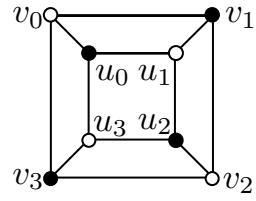

$Q_{2}$

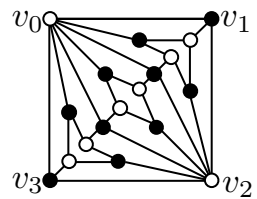

$Q_{2}^{(3)}$

Figure 5: Inside a quadrilateral region.

Lemma 4.3. Let $C=v_{0} v_{1} v_{2} v_{3}$ be a cycle of length 4 bounding a 2-cell region $D$ in a $\mathcal{P}$-irreducible quadrangulation $G$ of a non-spherical closed surface. Then, the interior of $D$ has one of the structures $Q_{1}$ and $Q_{2}^{(n)}(n \geq 1)$, as shown in Figure 5.

Proof. Use induction on the number of faces in $D$, say $F \geq 1$. If $F=1$, then it is clear that $D$ corresponds to a face of $G$ and it has the structure $Q_{1}$. Hence we suppose that $F \geq 2$ below.

If $G \cap \bar{D}$ is not an attached cube $Q_{2}$, then there is a vertex $x$ which is adjacent to both $v_{0}$ and $v_{2}$ (or $v_{1}$ and $v_{3}$ ) by Lemma 4.2. By the inductive hypothesis and Lemma 3.9, two quadrilateral regions bounded by $v_{0} v_{1} v_{2} x$ and $v_{2} v_{3} v_{0} x$ are filled with $Q_{2}^{(l)}$ and $Q_{2}^{(m)}$ for $n, m \geq 1$. As a result, we obtain $Q_{2}^{(n)}$ with $n=l+m$ and the induction is completed.

Note that replacing $Q_{2}$ with $Q_{2}^{(n)}$ having the same facing cube diagonal pair preserves the property being a $\mathcal{P}$-irreducible quadrangulation for any $n \geq 2$. Hence, there exist infinitely many $\mathcal{P}$-irreducible quadrangulations of a non-spherical closed surface $F^{2}$ if $F^{2}$ admits one with an attached cube. To avoid the complexity in figures, we use simply $Q_{2}$ to represent any $Q_{2}^{(n)}$ after this.

In the following lemmas, we discuss inside structures of regions bounded by 6 - and 8 -cycles. For brevity, we shall omit routines in the proofs.

Lemma 4.4. Let $C=v_{0} v_{1} v_{2} v_{3} v_{4} v_{5}$ be a trivial cycle of length 6 bounding a 2-cell region $D$ in a $\mathcal{P}$-irreducible quadrangulation $G$ of a non-spherical closed surface. Then, the interior of $D$ has one of the structures $H_{1}, H_{2}, \ldots, H_{17}$, as shown in Figure 6.

Proof. As well as the previous lemma, we use induction on the number of faces in $D$, say $F \geq 2$. If $F=2$, then $D$ has the structure $H_{1}$. Hence we suppose that $F \geq 3$. Observe that the existence of a short path of (i), (ii) or (iv) is guaranteed by Lemma 4.2. We fill the divided regions with pieces as follows.

If $C$ has a diagonal, then we apply Lemma 4.3 and obtain $H_{1}, H_{6}$ and $H_{10}$ in Figure 6. Further, if there is an inner vertex $x$ which is adjacent to both $v_{0}$ and $v_{2}$, then the quadrilateral region bounded by $x v_{0} v_{1} v_{2}$ is filled with $Q_{1}$ or $Q_{2}^{(n)}(n \geq 1)$, and the hexagonal region bounded by $v_{0} x v_{2} v_{3} v_{4} v_{5}$ is filled with $H_{i}$ for some $i \in\{1, \ldots, 17\}$ by the inductive hypothesis. Checking the whole cases is a routine, so we omit it, however, most cases are excluded by lemmas in Section 3.

Furthermore, assume that $D$ contains two inner vertices $x$ and $y$ such that 3-path $v_{0} x y v_{3}$ runs across $D$. Also in this case, we apply the inductive hypothesis to two separated hexagonal regions and obtain $H_{i}$ 's in Figure 6. 

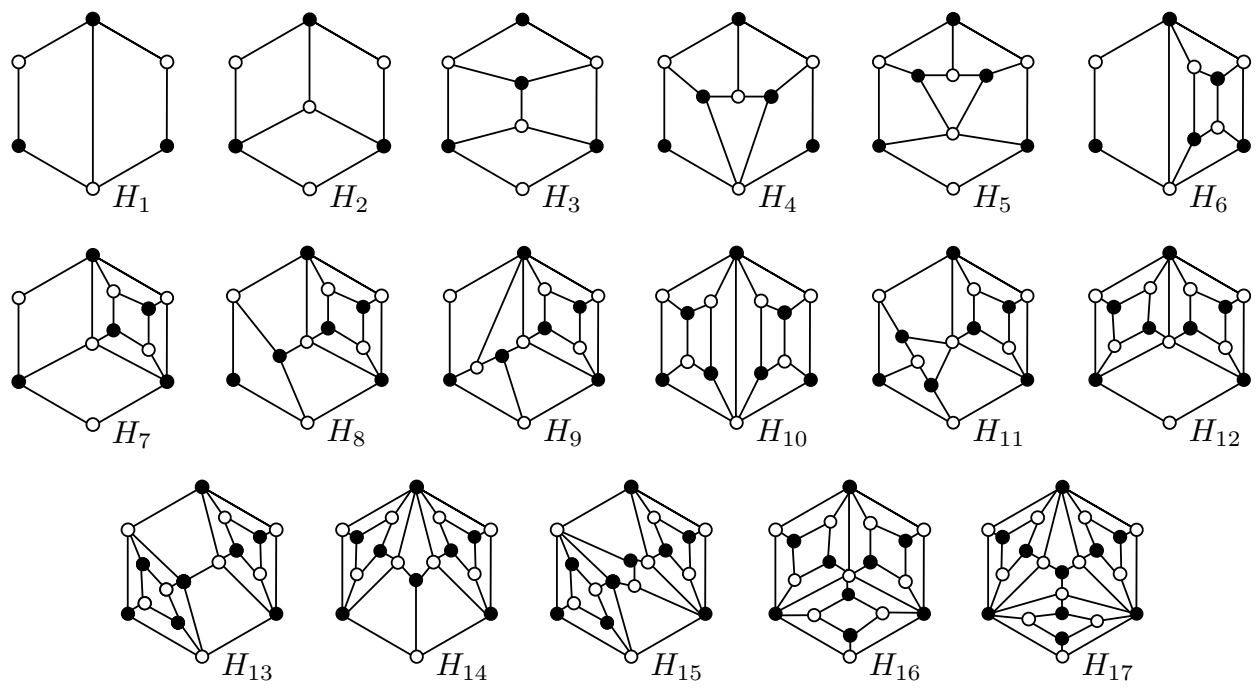

Figure 6: Inside a hexagonal region.

Lemma 4.5. Let $C=v_{0} v_{1} v_{2} v_{3} v_{4} v_{5} v_{6} v_{7}$ be a trivial cycle of length 8 bounding a 2-cell region $D$ in a $\mathcal{P}$-irreducible quadrangulation $G$ of a non-spherical closed surface. If $D$ has no diagonal edge and no attached cube, then the interior of D has one of the structures $\mathrm{O}_{1}, \mathrm{O}_{2}, \ldots, \mathrm{O}_{8}$, as shown in Figure 7.
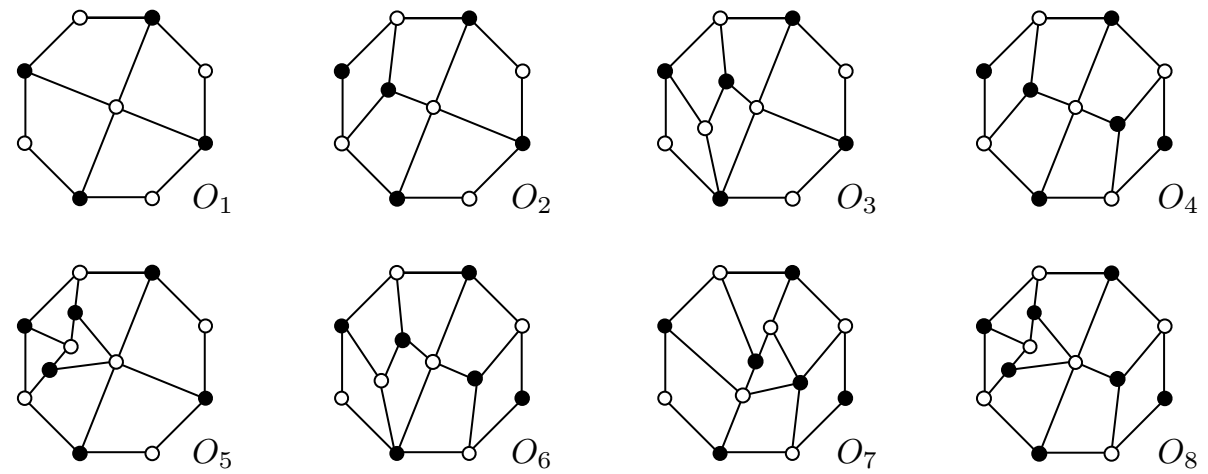

Figure 7: Inside an octagonal region.

Proof. In this proof, all subscripts of vertices are taken modulo 8 . We also use induction on the number of faces in $D$, say $F$. If $F$ is at most 3, then $D$ has a diagonal, contrary to the assumption of the lemma. If $F=4$, then $D$ includes a single vertex by Euler's formula and it should be adjacent to $v_{i}, v_{i+2}, v_{i+4}$ and $v_{i+6}$; for otherwise, $D$ would contain a diagonal. This is clearly $O_{1}$ in Figure 7 . Therefore, we assume $F \geq 5$ hereafter. Observe that $D$ contains a short path of type (ii), (iii) or (iv) by Lemma 4.2. 
First, we assume that $D$ includes an inner vertex $x$ which is adjacent to both $v_{0}$ and $v_{4}$. Then there are two hexagonal regions $D^{\prime}$ and $D^{\prime \prime}$ bounded by $x v_{0} v_{1} v_{2} v_{3} v_{4}$ and $x v_{4} v_{5} v_{6} v_{7} v_{0}$ respectively. Note that each of $D^{\prime}$ and $D^{\prime \prime}$ contains no attached cube. Then by the previous lemma, we fill them with $H_{1}, H_{2}, H_{3}, H_{4}$ and $H_{5}$ in Figure 6 so that the whole configuration satisfies the condition of this lemma. By considering lemmas in Section 3, most cases are excluded and we obtain $\mathrm{O}_{1}, \mathrm{O}_{2}, \mathrm{O}_{3}, \mathrm{O}_{4}, \mathrm{O}_{5}, \mathrm{O}_{6}$ and $\mathrm{O}_{8}$ in Figure 7. Therefore, after this, we suppose that $D$ contains no such vertex.

Secondly we assume that there is an inner vertex $x$ in $D$ which is adjacent to both $v_{0}$ and $v_{2}$. Then, there are a quadrilateral region $D^{\prime}$ and an octagonal region $D^{\prime \prime}$ divided by the 2-path $v_{0} x v_{2}$. By the assumption and Lemma $4.3, D^{\prime}$ bounds a face of $G$. If $D^{\prime \prime}$ contains a diagonal edge, then it should be $x v_{4}$ or $x v_{6}$ by Lemma 3.2. However, in each case, there would be a forbidden 2-path; e.g., $v_{0} x v_{4}$ if the diagonal $x v_{4}$ exists. Hence, we may assume that $D^{\prime \prime}$ contains no diagonal. Now we apply the inductive hypothesis and fill $D^{\prime \prime}$ with $O_{1}, \ldots, O_{8}$ in Figure 7 ; note that most cases would contain a contractible face or a shrinkable 2-path by lemmas in Section 3. As a result, we obtain $O_{1}, \ldots, O_{8}$. Then we also assume that $D$ does not include such a 2-path.

Finally, we assume that $D$ has a short path of type (iv) in Lemma 4.2. Actually, this 3-path divides $D$ into a hexagonal region and an octagonal one. As well as the above case, we use the inductive hypothesis and Lemma 4.4, and obtain our conclusion.

Lemma 4.6. Let $G$ be a $\mathcal{P}$-irreducible quadrangulation of the projective plane. If $G$ has a hexagonal 2-cell region $D$ such that $G \cap \bar{D}$ is isomorphic to either $H_{13}$ or $H_{15}$ in Lemma 4.4, then $G$ is one of $I_{1}, I_{2}$ and $I_{3}$ shown in Figure 8.

Proof. Let $C=v_{0} v_{1} v_{2} v_{3} v_{4} v_{5}$ be a 6 -cycle bounding a hexagonal region $D$ such that $G \cap \bar{D}$ is isomorphic to either $H_{13}$ or $H_{15}$. We may assume that each of $v_{0} v_{1} v_{2} x$ and $v_{3} v_{4} v_{5} y$ bounds $Q_{2}$ where $x$ and $y$ are distinct inner vertices of $D$. Now, cube diagonal pairs $\left\{v_{0}, v_{2}\right\}$ and $\left\{v_{3}, v_{5}\right\}$ are facing and there are such faces $f_{1}=v_{0} p v_{2} q$ and $f_{2}=v_{3} s v_{5} t$ outside of $D$ by Lemma 3.16, where $p, q, s, t \in V(G)$.

However, if $f_{1} \neq f_{2}$, the two essential diagonal (or semi-diagonal) curves in Lemma 3.16 do not exist together on the projective plane. Therefore, we have $f_{1}=f_{2}$, that is, $v_{0} v_{3}, v_{2} v_{5} \in E(G)$ and $f_{1}=f_{2}$ is bounded by $v_{0} v_{3} v_{2} v_{5}$. Under the conditions, the 6-cycle $v_{0} x v_{2} v_{5} y v_{3}$ bounds a 2-cell region and it should be filled with either $H_{13}$ or $H_{15}$ by Lemma 4.4. Actually we have three ways to take a pair $\left\{H_{i}, H_{j}\right\}$ for $i, j \in\{13,15\}$ and the lemma follows; for example, if we fill those hexagonal regions with two $H_{13}$ 's then we obtain $I_{1}$.

\section{Regions bounded by 6- or 8-walks}

A boundary walk of a hexagonal region of a $\mathcal{P}$-irreducible quadrangulation is not always a cycle, and the same vertex often appears twice along it. Such a hexagonal region can contain the following structure that generates an infinite series of $\mathcal{P}$-irreducible quadrangulations of a non-spherical closed surface.

Let $h_{1}, h_{2}$ and $h_{3}$ be three pieces with two terminals $x_{1}$ and $x_{2}$ shown in the first three configurations of Figure 9, and let $\left[s_{1}, \ldots, s_{m}\right]$ be a given sequence of 1,2 and 3 of any length such that each of 2 and 3 does not continue; i.e., we do not permit a sequence like $[\ldots, 2,2, \ldots]$. Put $h_{s_{1}}$ to $h_{s_{m}}$ in a hexagon $a_{1} b_{1} c a_{2} b_{2} d$ so that each $x_{i}$ coincides with $a_{i}$ for $i \in\{1,2\}$, and identify paths between $x_{1}$ and $x_{2}$ in each neighboring pair 

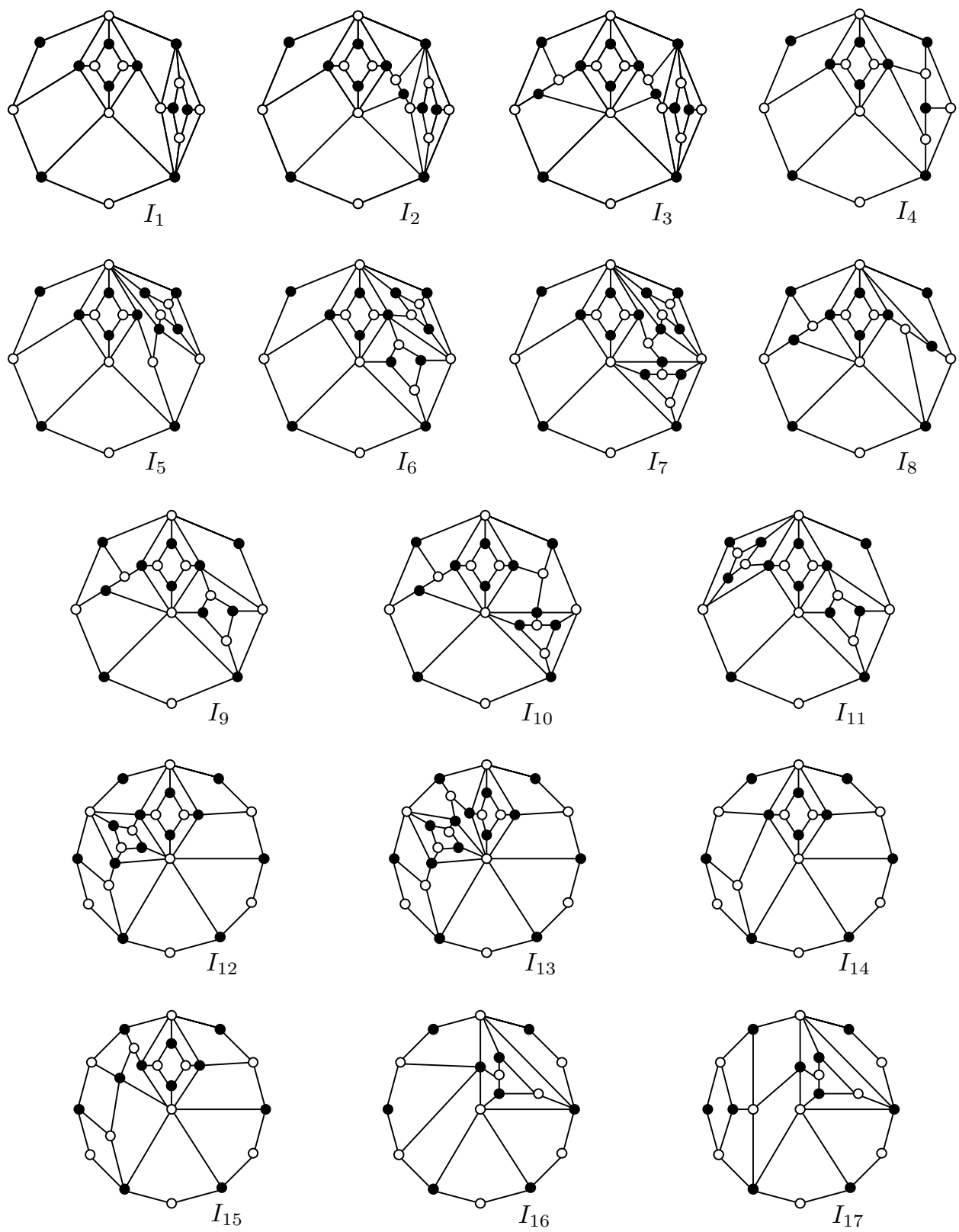

Figure 8: The 17 families of bipartite $\mathcal{P}$-irreducible quadrangulations with attached cubes. 
of pieces. (See the rightmost configuration of Figure 9.) We denote the resulting graph by $H_{18}\left[s_{1}, \ldots, s_{m}\right]$; note that we implicitly exclude $H_{18}[2]$ and $H_{18}[3]$ since they cannot fill the hexagonal region solely. If $H_{18}\left[s_{1}, \ldots, s_{m}\right]$ is contained in a $\mathcal{P}$-irreducible quadrangulation $G$ so that $a_{1}=a_{2}$, then each attached cube is not removable and each face is not contractible in this configuration; note that $G$ is nonbipartite. We often denote $H_{18}\left[s_{1}, \ldots, s_{m}\right]$ simply by $H_{18}$.
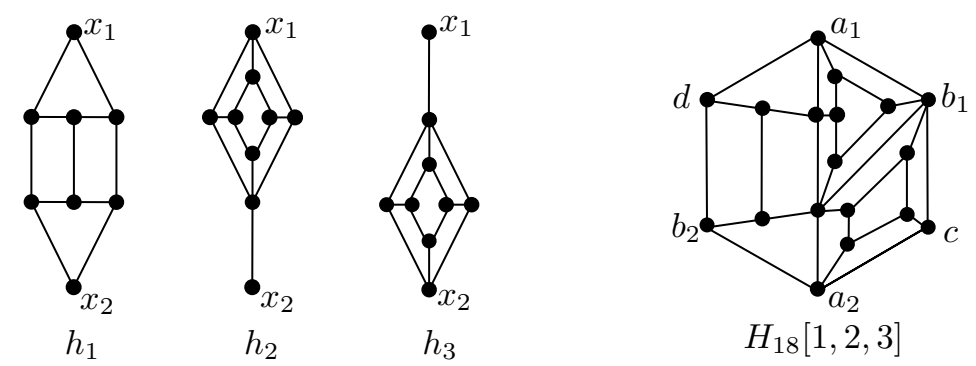

Figure 9: Inside a hexagonal region including an infinite series $H_{18}$.
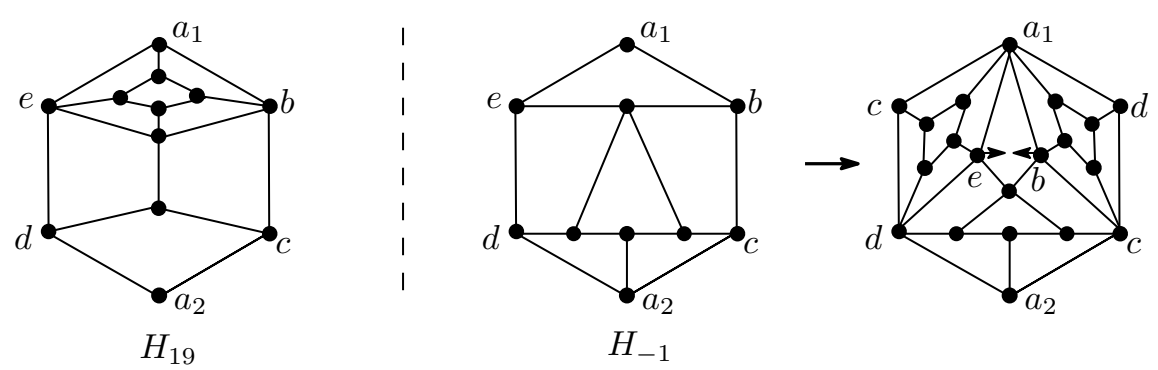

Figure 10: Inside a hexagonal region bounded by a closed walk (1).

See $H_{19}$ in Figure 10. Note that the hexagonal region is bounded by a closed walk $W=a_{1} b c a_{2} d e$ where $a_{1}=a_{2}(=a)$ and the other four vertices $b, c, d$ and $e$ are distinct. Actually, $H_{19}$ is appeared as a partial structure in $\mathcal{P}$-irreducible quadrangulations of the projective plane. (In Lemma 5.3, it will be mentioned.) However, the following lemma can exclude $H_{19}$ from the later arguments.

In the following three lemmas (Lemmas 5.1, 5.2 and 5.3), we let $D$ be a hexagonal region bounded by a closed walk $W=a_{1} b c a_{2} d e$ in a $\mathcal{P}$-irreducible quadrangulation $G$ of the projective plane where $a_{1}=a_{2}(=a)$ and the other four vertices $b, c, d$ and $e$ are distinct.

Lemma 5.1. If $G \cap \bar{D} \cong H_{19}$, then $G$ is isomorphic to $I_{20}$ in Figure 11 .

Proof. Note that $G$ is nonbipartite since $G$ contains an essential cycle of length 3 . Therefore, $G$ has an edge be outside of $D$ by Lemma 3.16. Then there are two quadrilateral regions bounded by abed and aebc. By Lemma 4.3, each of these regions is filled with either $Q_{1}$ or $Q_{2}$. However, if $Q_{1}$ is used, that is, it corresponds to a face of $G$, then we can 

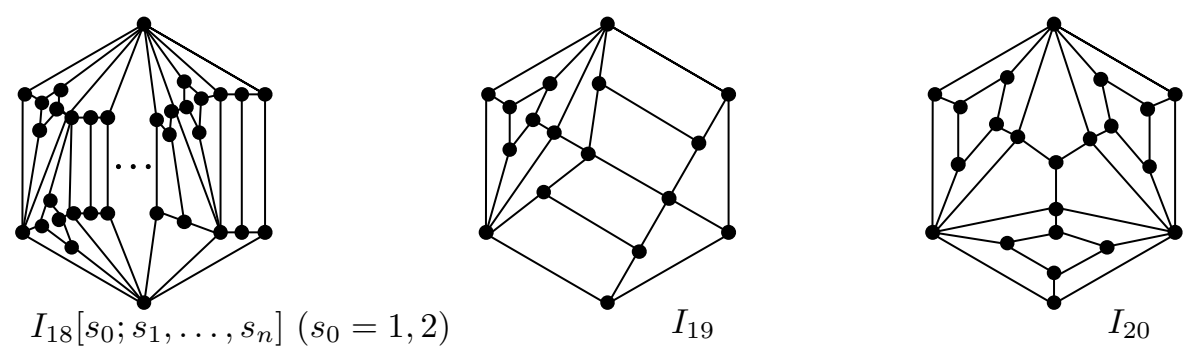

Figure 11: The 3 families of nonbipartite $\mathcal{P}$-irreducible quadrangulations.

easily find an essential simple closed curve intersecting $G$ at only two vertices, a contradiction. Hence, we fill each of those regions with $Q_{2}$ and obtain $I_{20}$ in Figure 11.

Lemma 5.2. $G \cap \bar{D}$ cannot be isomorphic to $H_{-1}$ in Figure 10 .

Proof. Similarly to Lemma 5.1, $G$ has an edge $c d$ in this case by Lemma 3.14, and both of two quadrilateral regions outside of $D$ are filled with $Q_{2}$ (see the right-hand side of Figure 10). However in this case, we would find a contractible face at $\{e, b\}$ by Lemmas 3.8 and 3.12. Therefore the lemma follows.

Lemma 5.3. $G \cap \bar{D}$ is isomorphic to either $H_{18}$ or $H_{19}$.

Proof. We use induction on the number of faces in $D$, say $F$. If $F$ is at most 3 , then $D$ includes at most one inner vertex by Euler's formula. In this case, although $\partial(D)$ is not a cycle, $G \cap \bar{D}$ forms a structure like either $H_{1}$ or $H_{2}$ in Figure 6; we have to identify the top and the bottom vertices of $H_{i}$ for $i \in\{1,2\}$. However, $G$ would have representativity at most 2 , a contradiction. (Such an essential simple closed curve passes through $a$.) If $F=4, D$ includes exactly two vertices and we have $G \cap \bar{D} \cong H_{18}[1]$. Therefore, we assume that $F \geq 5$ after this. Similarly to the former lemmas, we discuss inner structures of divided regions by a short path; we have to consider (i), (ii) and (iv) in Lemma 4.2.

First, we assume that $D$ contains a diagonal edge. By Lemma 3.2 and the simplicity of $G$, it should be $c e$ or $b d$, now say $c e$, up to symmetry. Then each of two quadrilateral regions bounded by $a_{1} b c e$ and $d a_{2} c e$ should be filled with $Q_{2}$; otherwise at least one of those regions forms a face of $G$, but we can easily find an essential simple closed curve passing through only two vertices of $G$. Therefore, we obtain $H_{18}[3,2]$ from this case. Then, we assume that $D$ contains no diagonal hereafter.

Secondly, we assume that $D$ includes an inner vertex $x$ which is adjacent to $a_{1}$ and $c$. Then the quadrilateral region $D^{\prime}$ bounded by $a_{1} b c x$ is filled with either $Q_{1}$ or $Q_{2}$. If we have the former, that is, $a_{1} b c x$ bounds a face of $G$, then we would find an essential simple closed curve intersecting $G$ only at $a$ and $c$, contrary to the assumption. Therefore, we assume the latter case. In this case, the hexagonal region $D^{\prime \prime}$ bounded by a 6 -walk $a_{1} x c a_{2} d e$ satisfies the assumption of this lemma. Thus, we use the inductive hypothesis and fill the region with either $H_{18}$ or $H_{19}$. If we use $H_{19}$, then the configuration becomes a part of $I_{20}$ by Lemma 5.1. However, this is not the case since $b$ corresponds to $d$. Hence we fill $D^{\prime \prime}$ with $H_{18}$ and obtain our desired conclusion. Then after this, we assume that there is no such inner vertex like $x$. (We also exclude similar paths $a_{1} x d, a_{2} x b$ and $a_{2} x e$.) 
Thirdly, we assume that there is an inner vertex $x$ which is adjacent to $b$ and $e$ (or $c$ and $d$ ). Then, there are a quadrilateral region bounded by $a_{1} b x e$ and a hexagonal region bounded by a 6 -cycle $b c a_{2} d e x$. We fill these regions by using the results of Lemmas 4.3 and 4.4 respectively. Most cases are excluded by lemmas in Section 3, but we obtain $H_{18}[1], H_{18}[3,2,3]$ and $H_{19}$ by filling them with $\left\{Q_{1}, H_{2}\right\},\left\{Q_{2}, H_{10}\right\}$ and $\left\{Q_{2}, H_{2}\right\}$, respectively. (For reference, when we use $\left\{Q_{1}, H_{4}\right\}$, we obtain $H_{-1}$ in Figure 10, but it had already been excluded by Lemma 5.2.)

Next, we consider the existence of an essential 3-cycle $a_{1} x y a_{2}$ where $x$ and $y$ are inner vertices of $D$. In this case, we can apply the inductive hypothesis and fill two hexagonal regions with $H_{18}$ 's and obtain our conclusion; we do not have to consider $H_{19}$ by Lemma 5.1.

Finally we assume that there is a 3-path bxyd (or cxye) where both $x$ and $y$ are inner vertices of $D$. Then the boundary of each hexagonal region divided by the 3 -path is a cycle, and we fill them by using Lemma 4.4. We only have to check $H_{i}$ for $i \in\{3,4,5\}$, since the existence of an attached cube, a diagonal edge and a single vertex of degree 3 clearly yields a short path discussed above. However, there is no pair to satisfy the conditions from this case. Hence, the induction is completed.

In the following lemma, we discuss a hexagonal region bounded by a 6 -walk in which two vertices each appear twice.

Lemma 5.4. Let $D$ be a hexagonal region bounded by a closed walk $W=a_{1} b_{1} c a_{2} b_{2} d$ in $a \mathcal{P}$-irreducible quadrangulation $G$ of the projective plane with $a_{1}=a_{2}(=a)$ and $b_{1}=b_{2}$ $(=b)$. Then $G \cap \bar{D}$ is isomorphic to one of $H_{18}, H_{20}$ and $H_{21}$.

Proof. Since almost the same argument of the previous proof holds, we omit the proof of this lemma. However, we should pay attention to the following points:

(1) When assuming that there is a 3-path cxyd where $x$ and $y$ are inner vertices of $D$, we obtain $\mathrm{H}_{20}$ in Figure 12; note that such a configuration was excluded in the previous lemma, since at least one of shaded faces in the right-hand side of Figure 12 is contractible by Lemma 3.8.

(2) If there is an essential 3-cycle $a_{1} x y a_{2}\left(\right.$ or $\left.b_{1} x y b_{2}\right)$, then we apply Lemma 5.3 to each of two hexagonal regions divided by the cycle.

(3) Using $Q_{2}$ and $H_{19}$, we can construct $H_{21}$ in Figure 12.

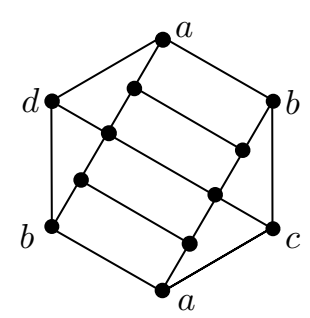

$H_{20}$

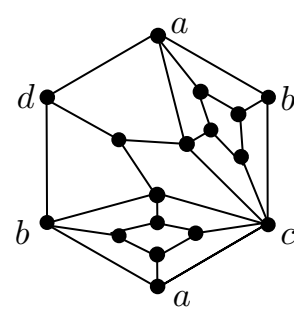

$H_{21}$

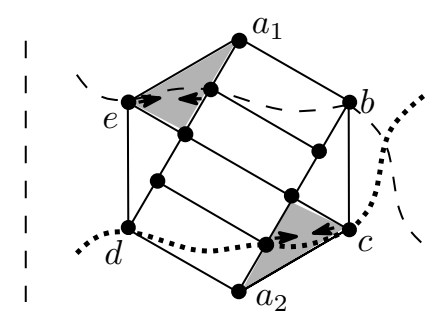

Figure 12: Inside a hexagonal region bounded by a closed walk (2). 


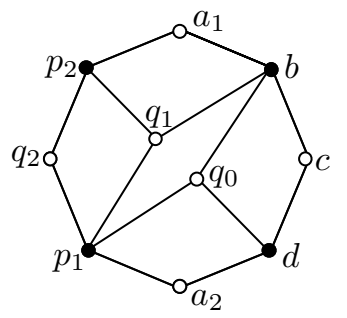

$\mathrm{O}_{9}(2)$

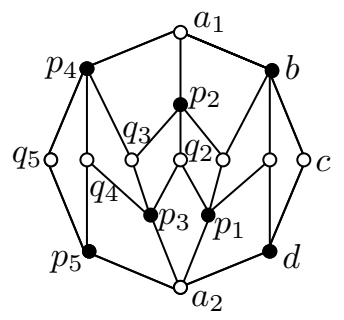

$\mathrm{O}_{9}(5)$

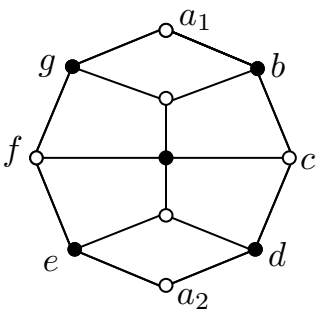

$\mathrm{O}_{10}$

Figure 13: Octagonal structure generating infinite series.

See the graph denoted by $O_{9}(2)$ shown in the left-hand side of Figure 13. Observe that the octagonal region $D$ is bounded by a closed walk $W=a_{1} b c d a_{2} p_{1} q_{2} p_{2}$ where $a_{1}=a_{2}(=a)$ and the other vertices are distinct. Now add two vertices $p_{3}$ and $q_{3}$ so that $q_{2} p_{1} a_{2} p_{3}$ and $p_{2} q_{2} p_{3} q_{3}$ are quadrilateral faces. The resulting graph is denoted by $O_{9}(3)$. We inductively define the general form $O_{9}(m)$ from $O_{9}(m-1)$ by adding two vertices $p_{m}$ and $q_{m}$ so that $q_{m-1} p_{m-2} a_{2} p_{m}$ and $p_{m-1} q_{m-1} p_{m} q_{m}$ (resp., $a_{1} p_{m-2} q_{m-1} p_{m}$ and $\left.p_{m} q_{m-1} p_{m-1} q_{m}\right)$ are quadrilateral faces if $m$ is odd (resp., even); note that we define $O_{9}(m)$ for $m \geq 2$. This $O_{9}(m)$ satisfies the followings:

(a) $\operatorname{deg}\left(q_{i}\right)=3$ for each $i \in\{0, \ldots, m-1\}$, while $\operatorname{deg}\left(p_{i}\right)=4$ for each $i \in$ $\{1, \ldots, m-2\}$ if $m \geq 3$.

(b) If $m$ is odd, then $\operatorname{deg}_{D}(b)=2, \operatorname{deg}_{D}(c)=0, \operatorname{deg}_{D}(d)=1, \operatorname{deg}_{D}\left(p_{m}\right)=1$, $\operatorname{deg}_{D}\left(q_{m}\right)=0$ and $\operatorname{deg}_{D}\left(p_{m-1}\right)=2$.

(c) If $m$ is even, then $\operatorname{deg}_{D}(b)=2, \operatorname{deg}_{D}(c)=0, \operatorname{deg}_{D}(d)=1, \operatorname{deg}_{D}\left(p_{m-1}\right)=2$, $\operatorname{deg}_{D}\left(q_{m}\right)=0$ and $\operatorname{deg}_{D}\left(p_{m}\right)=1$.

Lemma 5.5. Let $D$ be an octagonal region bounded by a closed walk $W=a_{1} b c d a_{2}$ efg in a $\mathcal{P}$-irreducible quadrangulation $G$ of the projective plane such that $a_{1}=a_{2}(=a)$ and the other vertices are distinct. Suppose the following conditions hold:

( $\alpha$ ) Each of $\operatorname{deg}_{D}(b), \operatorname{deg}_{D}(d), \operatorname{deg}_{D}(e)$ and $\operatorname{deg}_{D}(g)$ is at least 1.

( $\beta$ ) No two vertices of degree 3 in $D$ are adjacent.

Then $G \cap \bar{D}$ is isomorphic to either $O_{9}(m)$ or $O_{10}$ in Figure 13.

Proof. First of all, we show that $D$ contains no diagonal edge. Suppose to the contrary that there is a diagonal edge in $D$, say $b f$; note that a diagonal edge like $a_{1} d$ is immediately excluded since it yields multiple edges. Then, there is a quadrilateral region $D^{\prime}$ bounded by a 4 -cycle $a_{1} b f g$. By Lemma 4.3 and the condition $(\beta)$ in the lemma, $D^{\prime}$ should be filled with $Q_{1}$, that is $D^{\prime}$ corresponds to a face of $G$. However, it contradicts $(\alpha)$ in the lemma. Therefore, we conclude that $D$ has no diagonal.

Now, we use induction on the number of faces in $D$, say $F$ as well as previous lemmas. If $F$ is at most 4, then $D$ includes at most one inner vertex $x$ by Euler's formula. Since $D$ has no diagonal, $G \cap \bar{D}$ is a graph obtained from $O_{1}$ in Figure 7 by identifying a pair of 
antipodal vertices. However in this case, we would find an essential simple closed curve intersecting $G$ at only $\{a, x\}$, a contradiction. By careful observation, we have the unique configuration $O_{9}(2)$ with $F=5$ faces in $D ; D$ includes exactly two inner vertices of degree 3 . Therefore, the first step of the induction holds.

Similarly to the former lemmas, we divide the following argument along Lemma 4.2; other than (i) which is already excluded. Note that we shall implicitly exclude a short path already discussed in the former arguments.

Case I. There exists a short 2-path (ii) or (iii): First, such a vertex $x$ adjacent to $a_{1}$ and $c$ violates condition $(\alpha)$ in the lemma, since $D$ does not contain an attached cube by $(\beta)$. (We also exclude such 2-paths $a_{1} x f, a_{2} x c$ and $a_{2} x f$.) Therefore, we assume that there is such a vertex $x$ adjacent to $b$ and $d$. Then the 2-path $b x d$ divides $D$ into an octagonal region $D^{\prime}$ and a quadrilateral region $D^{\prime \prime}$; note that $D^{\prime \prime}$ corresponds to a face of $G$. If $\operatorname{deg}_{D^{\prime}}(b), \operatorname{deg}_{D^{\prime}}(d) \geq 1$, then we can apply the inductive hypothesis. However, if we use $O_{9}(m)$, then $x$ would become degree 2 . On the other hand, if we fill $D^{\prime}$ with $O_{10}$, then the face-contraction of $b c d x$ at $\{c, x\}$ can be applied by Lemma 3.8. If $\operatorname{deg}_{D^{\prime}}(b)=0$ and $\operatorname{deg}_{D^{\prime}}(d)=0$, then we can easily find an essential simple closed curve intersecting $G$ at only $a$ and $x$; we can take such a curve along $a_{1} b x d a_{2}$.

Therefore, we assume that one of $\operatorname{deg}_{D^{\prime}}(b)$ and $\operatorname{deg}_{D^{\prime}}(d)$ is equal to 0 and the other is at least 1 . We may assume that $\operatorname{deg}_{D^{\prime}}(b)=0$ and $\operatorname{deg}_{D^{\prime}}(d) \geq 1$ without loss of generality. Under the condition, there is a face of $G$ in $D^{\prime}$ bounded by $a_{1} b x y$ for $y \in V(G)$. If $y$ is a vertex of $W$, then we have either $y=e$ or $y=g$ by Lemma 3.2. If we assume the former, then there would be multiple edges $a e$, contrary to our assumption. On the other hand, if the latter holds, there is a hexagonal region $D^{\prime \prime \prime}$ bounded by a cycle $d a_{2}$ efgx of $G$. By Lemma 4.4 and the condition $(\beta), D^{\prime \prime \prime}$ is filled only with $H_{2}$ and we obtain $O_{9}(2)$; the unique inner vertex of degree 3 in $D^{\prime \prime \prime}$ must have neighbors $\{d, e, g\}$, otherwise, $(\alpha)$ cannot be held.

Therefore we may suppose that $y$ is an inner vertex of $D^{\prime}$. In this case, the octagonal region $D^{*}$ bounded by $a_{1} y x d a_{2} e f g$ satisfies the conditions of this lemma and hence we can apply the inductive hypothesis to $D^{*}$; observe that $\operatorname{deg}_{D^{*}}(y) \geq 1$. Under our assumptions, we fill $D^{*}$ with $O_{9}(m)$ so as not to have adjacent vertices of degree 3 , and obtain $\mathrm{O}_{9}(m+1) ; \mathrm{O}_{10}$ is inappropriate since it yields two adjacent vertices of degree 3 in $D$.

Next, we assume that there is an inner vertex $x$ of $D$ adjacent to both of $b$ and $g$. Let $D^{\prime}$ be an octagonal region bounded by $b c d a_{2}$ efgx; note that the 4-cycle $a_{1} b x g$ bounds a face of $G$. If $D^{\prime}$ has a diagonal edge, then the one end should be $x$ since $D$ admits no diagonal. However, if there is such a diagonal, say $x d$, then there would be a forbidden 2-path $b x d$, which was already discussed above. Thus $D^{\prime}$ has no diagonal edge and we can apply Lemma 4.5 to the region. In fact, most cases are excluded by some conditions but we obtain $\mathrm{O}_{9}(3)$ and $\mathrm{O}_{10}$ by using $\mathrm{O}_{4}$ and $\mathrm{O}_{2}$, respectively. By the similar argument as above, we obtain $O_{9}(2)$ (resp., $O_{10}$ ) if we assume that there is a 2-path bxe (resp., $c x f$ ) for an inner vertex $x$ of $D$.

Case II. There exists a short 3-path (iii): First, assume that such a short 3-path is $a_{1} x y d$ where $x$ and $y$ are inner vertices of $D$. In this case, the hexagonal 2-cell region $D^{\prime}$ bounded by $a_{1} x y d c b$ should be filled with either $H_{1}$ or $H_{2}$ by Lemma 4.4 and the condition $(\beta)$ in this lemma. However in each case, $D$ would contain a diagonal or a forbidden 2-path excluded by the above arguments. By the same reason, we do not have to consider a 3-path like bxyf. (Of course, we exclude the paths of the same type, considering the symmetry; 
e.g., $\left.a_{2} x y g.\right)$

Case III. There exists a short 4-cycle (iv): We assume that there exists an essential 4-cycle $a_{1} x y z a_{2}$ for inner vertices $x, y$ and $z$ of $D$. Then, $D$ is divided into two octagonal regions $D^{\prime}$ and $D^{\prime \prime}$ and they are bounded by $a_{1} x y z a_{2} d c b$ and $a_{1} x y z a_{2} e f g$, respectively. Here, we consider degrees of $x$ and $z$ and first, suppose that $\operatorname{deg}_{D^{\prime}}(x)=0$. In this case, $D^{\prime}$ contains a face bounded by $a_{1} x y w$ for $w \in V(G)$. (Note that $\operatorname{deg}_{D^{\prime}}(z) \geq 1$ and $\operatorname{deg}_{D^{\prime \prime}}(z) \geq 1$; otherwise there would be an essential simple closed curve passing through only $a$ and $y$. Also, $\operatorname{deg}_{D^{\prime \prime}}(x)$ is clearly at least 1 .) The vertex $w$ is an inner vertex of $D^{\prime}$ since if not, $D$ would have a diagonal or a forbidden 3-path by the above argument. Let $D^{\prime \prime \prime}$ be the octagonal region bounded by $a_{1} w y z a_{2} d c b$; note that $\operatorname{deg}_{D^{\prime \prime \prime}}(w) \geq 1$. Then both of $D^{\prime \prime}$ and $D^{\prime \prime \prime}$ satisfy the inductive hypothesis and we fill them with $O_{9}(m)$ or $O_{10}$ so as not to make two adjacent vertices of degree 3 in $D$. Under the conditions, we only obtain $O_{9}(l)$ if $D^{\prime \prime}$ and $D^{\prime \prime \prime}$ are filled with $O_{9}\left(l^{\prime \prime}\right)$ and $O_{9}\left(l^{\prime \prime \prime}\right)$ respectively, where $l=l^{\prime \prime}+l^{\prime \prime \prime}+1$.

Therefore, we may assume that each of $\operatorname{deg}_{D^{\prime}}(x), \operatorname{deg}_{D^{\prime \prime}}(x), \operatorname{deg}_{D^{\prime}}(z)$ and $\operatorname{deg}_{D^{\prime \prime}}(z)$ is at least 1 . Then we also use the inductive hypothesis into $D^{\prime}$ and $D^{\prime \prime}$. However, every case is inappropriate, since using $O_{10}$ yields contractible face by Lemmas 3.8 and 3.12 and using two $O_{9}(m)$ 's makes $y$ to have degree 2 . Thus, the lemma follows.

\section{Classification by attached cubes}

Let $G$ be a $\mathcal{P}$-irreducible quadrangulation of the projective plane. Assume that $G$ has an attached cube $H$ with $\partial(H)=v_{0} v_{1} v_{2} v_{3}$ and an attached 4-cycle $C=u_{0} u_{1} u_{2} u_{3}$ such that $u_{i} v_{i} \in E(G)$ for each $i \in\{0,1,2,3\}$. Now, observe that any essential cycle of bipartite quadrangulations of the projective plane has even length while that of nonbipartite quadrangulations has odd length. This means that

(I) $G$ has an essential diagonal 3-curve $\gamma$ if $G$ is bipartite or

(II) $G$ has an essential semi-diagonal 3-curve $\gamma$ if $G$ is nonbipartite, such that $\gamma$ passes through $\left\{v_{0}, u_{1}, v_{2}\right\}$ by Lemma 3.16 .

First, we consider the case (I). In this case, $\gamma$ is passing through three faces $f_{1}=$ $v_{0} u_{0} u_{1} v_{1}, f_{2}=v_{1} u_{1} u_{2} v_{2}$ and $f=v_{0} a v_{2} b$ for $a, b \in V(G)$. Since $G$ is $\mathcal{P}$-irreducible, applying the face-contraction of $f$ at $\{a, b\}$ breaks the property. However, each of $\operatorname{deg}\left(v_{0}\right)$ and $\operatorname{deg}\left(v_{2}\right)$ is clearly at least four and hence we do not have to consider the 3-connectedness of the graph by Lemma 3.12. Thus, we further divide it into the following two cases:

(I-a) The face-contraction of $f$ disturbs the simplicity of the graph.

(I-b) The face-contraction of $f$ yields a quadrangulation with representativity at most 2 .

In (I-a), there exists a vertex $x$ adjacent to both $a$ and $b$ such that the 4 -cycle $C^{\prime}=v_{0} a x b$ is essential on the projective plane by Lemma 3.10. In this case, we cut the projective plane along $C^{\prime}$ and obtain (A) in Figure 14. In (I-b), $G$ has a diagonal 3-curve passing through $\{a, b, x\}$ and three faces $f, f^{\prime}=a c x d$ and $f^{\prime \prime}=b c^{\prime} x d^{\prime}$ by Lemma 3.8. Considering the identification of vertices except $u_{i}$ for $i \in\{1, \ldots, 4\}$, we obtain (B), (C) and (D) in Figure 14 up to symmetry; we have to pay attention to the simplicity, the degree conditions of the graph and Lemma 4.3, further and that it does not have the structure of (I-a). (For example, if $d^{\prime}=v_{2}$ in (B), then we have (C). Furthermore, if $x=v_{3}$ in (B), then $d^{\prime}$ (resp., 


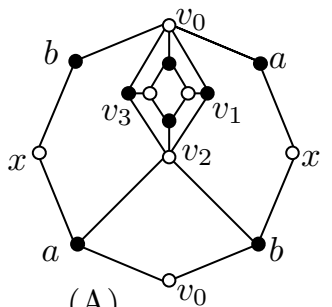

(A)

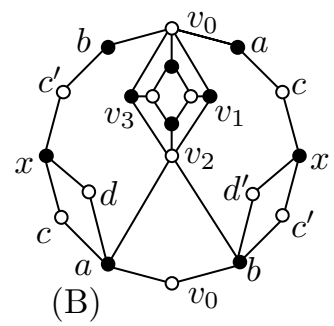

(B)

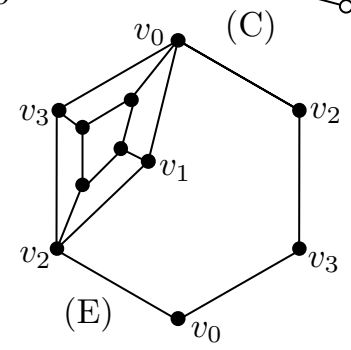

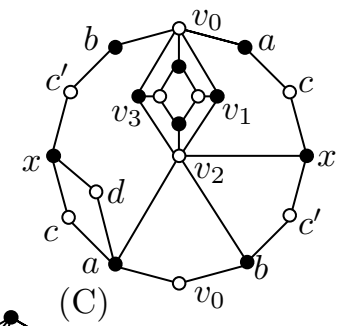

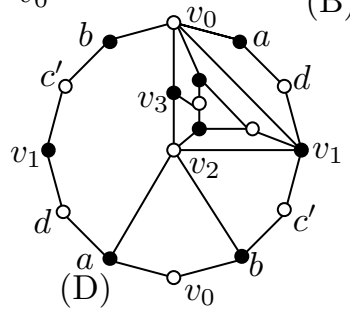

Figure 14: Around an attached cube.

d) must also coincide with $v_{2}$ (resp., c) by Lemma 4.3 and hence we obtain (D) in the figure. It is not so difficult to confirm that they are all.)

Secondly, we assume the case (II). In this case, $G$ has an essential semi-diagonal 3curve passing through $\left\{v_{0}, u_{1}, v_{2}\right\}$, that is, there is an edge joining $v_{0}$ and $v_{2}$. We cut open the projective plane along the essential 3-cycle $v_{0} v_{1} v_{2}$ and obtain $(\mathrm{E})$ in the figure.

In the first half of the next section, we determine $\mathcal{P}$-irreducible quadrangulations of the projective plane with attached cubes by filling each blank non-quadrilateral region of (A) to (E) with results in Sections 4 and 5.

\section{Proof of the main theorem}

We shall classify $\mathcal{P}$-irreducible quadrangulations of the projective plane in this section to prove Theorem 1.1, using the lemmas proved in the former sections. For our purpose, we divide our main result into the following four theorems, depending on the existence of an attached cube and bipartiteness.

Theorem 7.1. Let $G$ be a bipartite $\mathcal{P}$-irreducible quadrangulation of the projective plane. If $G$ has an attached cube, then $G$ is one of the graphs shown in Figure 8.

Proof. By the argument in the previous section, we first fill the two non-quadrilateral regions of (A) shown in Figure 14 with $H_{1}, \ldots, H_{17}$ so as to form a $\mathcal{P}$-irreducible quadrangulation. (However, we implicitly exclude $H_{13}$ and $H_{15}$ by Lemma 4.6.) In fact, we consider the hexagonal regions bounded by $v_{0} v_{1} v_{2} a x b$ and $v_{0} v_{3} v_{2} b x a$ and fill them with $H_{7}, H_{8}, H_{9}, H_{11}, H_{12}, H_{14}, H_{16}$ and $H_{17}$ since we have $\left\{v_{1}, v_{3}\right\} \cap\{a, b\}=\emptyset$; otherwise, $G$ would have multiple edges. When putting a pair of such pieces, we have to check the polyhedrality of $G$, and the absence of contractible face, removable 4-cycle and shrinkable 2-path, by using Proposition 3.1 and Lemmas 3.8, 3.9, 3.12-3.16.

Checking all the cases is a routine, and hence we present two bad examples below. First, see (i) of Figure 15, which is filled with a pair $\left(H_{7}, H_{9}\right)$. However, it is easy to see that this graph has representativity 2 . Secondly, see (ii) in the figure with a pair $\left(H_{11}, H_{12}\right)$. 
In this case, we can easily find a removable 4-cycle by Lemma 3.16, which is presented as the shaded region in the figure. Similarly to the above two bad cases, we can exclude almost pairs.

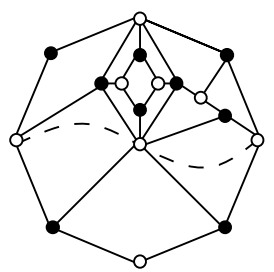

(i)

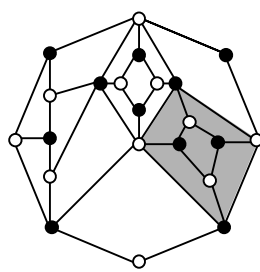

(ii)

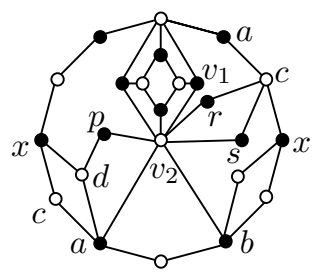

(iii)

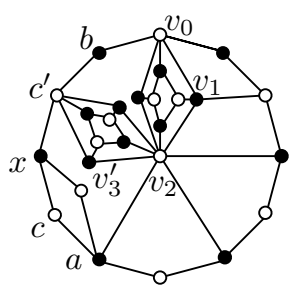

(iv)

Figure 15: Configurations in the proof of Theorem 7.1.

As a result, 8 pairs $\left(H_{7}, H_{11}\right),\left(H_{7}, H_{14}\right),\left(H_{7}, H_{16}\right),\left(H_{7}, H_{17}\right),\left(H_{9}, H_{9}\right),\left(H_{9}, H_{12}\right)$, $\left(H_{9}, H_{14}\right)$ and $\left(H_{12}, H_{12}\right)$ are available and we obtain $I_{4}, I_{5}, I_{6}, I_{7}, I_{8}, I_{9}, I_{10}$ and $I_{11}$ in Figure 8, respectively.

Next, we consider (B) in Figure 14. Consider the face-contraction of the face bounded by $x d a c$ at $\{c, d\}$. Observe that we have no identification of vertices $c, d, c^{\prime}$ and $d^{\prime}$ to other white vertices. (It was already done in the previous section.) Thus, we have that $\operatorname{deg}(x) \operatorname{deg}(a) \geq 4$. By Lemma 3.12, the face-contraction breaks the simplicity or the property of representativity at least 3 . It is easy to see that the former does not happen and hence we suppose the latter. That is, there is a diagonal 3-curve passing through either $\left\{c, d, v_{2}\right\}$ or $\left\{c, d, v_{0}\right\}$.

Assume that the curve passes the $\left\{c, d, v_{2}\right\}$ and other two faces $f_{1}$ and $f_{2}$ are bounded by $d p v_{2} q$ and $v_{2} r c s$ respectively, for $p, q, r, s \in V(G)$. (Actually, by Lemma 4.3, one of $p$ and $q$, say $q$, coincides with $a$. See (iii) in Figure 15.) If $s=x$ in the figure, then it would yield the configuration (C) in the Figure 14; we discuss (C) next. Further, if $s=b$, then the vertex $c$ is adjacent to both of $a$ and $b$ and hence it would become (A); it was already discussed. Moreover, if $r=a$, we would have multiple edges $v_{2} a$. Therefore, we can conclude that the unique possibility of the identification of such vertices is that $r=v_{1}$; note that we have considered all the possibility around $f_{2}$, since $G$ is bipartite and both of $r$ and $s$ should be black vertices. However, regardless of the unique identification, we can apply the face-contraction of $f_{2}$ at $\{r, s\}$ since there is no diagonal 3-curve passing through $r$ and $s$. This is contrary to $G$ being $\mathcal{P}$-irreducible. By the similar argument, we can find a contractible face when assuming that the diagonal 3-curve passes through $\left\{c, d, v_{0}\right\}$. As a result, (B) cannot be extended to any $\mathcal{P}$-irreducible quadrangulation.

As the third case, we consider (C) in Figure 14. By Lemma 3.16, there is no attached cube in the hexagonal region $D$ bounded by $v_{0} a c x v_{2} v_{1}$. Therefore, we try to put $H_{1}, \ldots, H_{5}$ into $D$. However, by Proposition 3.1 and Lemmas 3.9, 3.15 and 4.3, it is easy to confirm (but routine) that only $H_{1}$ is available and we have edge $v_{1} c$ in $D$.

Next, we consider the octagonal region $D^{\prime}$ bounded by $v_{0} v_{3} v_{2} a d x c^{\prime} b$. Assume that $D^{\prime}$ contains another attached cube $A$ such that $\partial(A)=v_{0}^{\prime} v_{1}^{\prime} v_{2}^{\prime} v_{3}^{\prime}$. Then its one cube diagonal pair, now say $\left\{v_{0}^{\prime}, v_{2}^{\prime}\right\}$, coincides with either $\{a, b\}$ or $\left\{c^{\prime}, v_{2}\right\}$ since it should be facing by Lemma 3.16. If the former occurs, then it clearly causes $I_{1}, I_{2}$ or $I_{3}$ by Lemmas 4.4 and 4.6. Thus, we suppose the latter (see (iv) of Figure 15). Now we fill the two hexagonal regions 
$H$ and $H^{\prime}$ bounded by $v_{0} v_{1} v_{2} v_{3}^{\prime} c^{\prime} b$ and $v_{2} a c x c^{\prime} v_{1}^{\prime}$, respectively. However, $H$ admits only $H_{12}$ and $H_{14}$, and $H^{\prime}$ does only $H_{8}$ by considering their partial structures. Therefore, we obtain $I_{12}$ and $I_{13}$ in Figure 8 in this case.

Then, we consider the possibility of existence of diagonal edges in the octagonal region $D^{\prime}$ and conclude that either $v_{3} c^{\prime}$ or $v_{3} d$ is available by some lemmas and $\mathcal{P}$-irreducibility. If both of diagonals are taken as edges of $G$, then $G$ becomes $I_{14}$. If one of two diagonals, say $v_{3} c^{\prime}$, is used, then we find the hexagonal region $H$ bounded by $c^{\prime} v_{3} v_{2} a c x$; note that $H$ does not contain its diagonal. We put $H_{2}, H_{3}, H_{4}$ and $H_{5}$ into $H$, however each of the resulting graphs has a contractible face; it is actually reducible into $I_{14}$. Furthermore, the same argument works when we use the diagonal $v_{3} d$.

Therefore we may assume that $D^{\prime}$ in (C) contains no attached cube and no diagonal, that is, it satisfies the condition of Lemma 4.5. Now, we try to put $O_{j}$ for $j \in\{1, \ldots, 8\}$ into $D^{\prime}$. Considering some lemmas in Section 3, we have only $I_{15}$ from this case by filling it with $\mathrm{O}_{2}$ in Figure 7.

Similarly to $(\mathrm{C})$, we consider the inside of octagonal region $O$ bounded by cycle $v_{0} v_{3} v_{2} a d v_{1} c^{\prime} b$ in the case (D). However, the argument is almost the same as the previous one and just a routine and hence we omit it here. (We first discuss the existence of an attached cube and diagonals in $O$. Next, we put the configurations of Figure 7.) As a result, we obtain $I_{16} I_{17}$ from (D). Therefore, the theorem follows.

We define $I_{18}\left[2 ; s_{1}, \ldots, s_{n}\right]$ as a graph obtained from (E) in Figure 14 by putting $H_{18}\left[s_{1}, \ldots, s_{n}\right]$ inside the hexagonal region. Recall that we forbid $I_{18}[2 ; \ldots, 2,2, \ldots]$, $I_{18}[2 ; \ldots, 3,3, \ldots], I_{18}[2 ; 2, \ldots]$ and $I_{18}[2 ; \ldots, 3]$, since we make it a rule to unify consecutive $Q_{2}$ 's to one.

Theorem 7.2. Let $G$ be a nonbipartite $\mathcal{P}$-irreducible quadrangulation of the projective plane. If $G$ has an attached cube, then $G$ is one of $I_{18}\left[2 ; s_{1}, \ldots, s_{n}\right], I_{19}$ and $I_{20}$ shown in Figure 11.

Proof. By the argument in the previous section, we have (E) in Figure 14 in this case. There is the unique blank hexagonal region $D$ which satisfies the conditions of Lemma 5.4. Hence, we fill $D$ with $H_{18}\left[s_{1}, \ldots, s_{n}\right]$ (resp., $H_{20}$ ) and obtain $I_{18}\left[2 ; s_{1}, \ldots, s_{n}\right]$ (resp., $I_{19}$ ); note that $H_{21}$ was already discussed in Lemma 5.1 and we obtained $I_{20}$.

In fact, some of $I_{18}\left[2 ; s_{1}, \ldots, s_{n}\right]$ with short sequences cannot satisfy the polyhedrality, hence we should exclude such "bad" sequences, which are listed in Table 1. It is not difficult to confirm that if $n \geq 4$, then any $I_{18}\left[2 ; s_{1}, \ldots, s_{n}\right]$ satisfying the above rule is acceptable. (Observe that there are different sequences $\left[s_{1}, \ldots, s_{n}\right] \neq\left[s_{1}^{\prime}, \ldots, s_{n}^{\prime}\right]$ such that $I_{18}\left[2 ; s_{1}, \ldots, s_{n}\right] \cong I_{18}\left[2 ; s_{1}^{\prime}, \ldots, s_{n}^{\prime}\right] ;$ e.g., $I_{18}[2 ; 1,1,2] \cong I_{18}[2 ; 3,1,1]$ in the table.)

Figure 16 presents six bipartite $\mathcal{P}$-irreducible quadrangulations of the projective plane without attached cubes. In the figure, $I_{26}(2 n+1)(n \geq 2)$ represents an infinite series of such graphs. The center white vertex of $I_{26}(2 n+1)$ has degree $2 n+1$ and each its black neighbors has degree 4 . Furthermore, it has $2 n+1$ vertices of degree 3 on the essential simple closed curve drawn by dotted circle. (We obtain the projective plane by identifying all pairs of antipodal points of the dotted circle.) In fact, the figure represents $I_{26}(7)$ with 15 vertices.

Theorem 7.3. Let $G$ be a bipartite $\mathcal{P}$-irreducible quadrangulation of the projective plane. If $G$ has no attached cube, then $G$ is one of the graphs shown in Figure 16. 
Table 1: Good and bad sequences for $\left[s_{1}, \ldots, s_{n}\right](n \leq 3)$.

$\left[s_{1}, \ldots, s_{n}\right](n \leq 3)$

$[1]$

$[1,1],[3,2]$

$[1,2],[3,1]$ bad (rep. 2)

good

bad (rep. 2)

good

Proof. For brevity, we write only the outline of the proof. We divide the proof into the following three cases by Lemmas 3.5, 3.13 and 3.15. Note that we prove those cases in this order, that is, we implicitly exclude a graph already appeared in the former cases.

Case I. $G$ has a 2-path $u_{0} u_{1} u_{2}$ induced by three vertices of degree 3: See (i) in Figure 17. In the figure, each antipodal pair of points of the dotted circle should be identified to obtain the projective plane. Note that $v_{0} v_{1} v_{2} v_{3} v_{4} v_{5}$ is a cycle of $G$ since if $v_{3}=v_{5}$, then $\operatorname{deg}\left(v_{4}\right)=3$ and $G$ would contain an attached cube.

The 2-path $u_{0} u_{1} u_{2}$ is not shrinkable and hence we have a face $v_{0} b v_{2} b^{\prime}$ by Lemma 3.14. Furthermore, we consider the face-contraction of the face $v_{2} v_{3} v_{4} u_{2}$ at $\left\{v_{3}, u_{2}\right\}$. Since $\operatorname{deg}\left(v_{2}\right), \operatorname{deg}\left(v_{4}\right) \geq 4$, we do not have to pay attention to the connectivity of the resulting graph by Lemma 3.12. Also, since $u_{1}$ is an inner vertex of the hexagon, the face-contraction preserves the simplicity of the graph. Hence, by Lemma 3.8, we have a face $v_{3} c v_{1} c^{\prime}$. By the same way, we find a face $v_{5} a v_{1} a^{\prime}$ (see the figure again).

Similarly to the argument in Section 6 and the previous theorem, we consider the possibility of identification of vertices and fill blank non-quadrilateral regions with $H_{1}, \ldots, H_{5}$ in Lemma 4.4. As a result, we obtain $I_{21}, I_{22}$ and $I_{23}$ from this case.
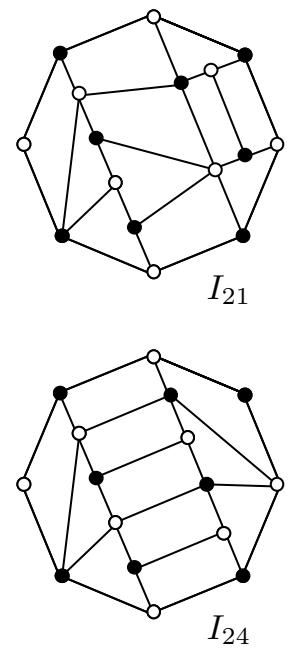
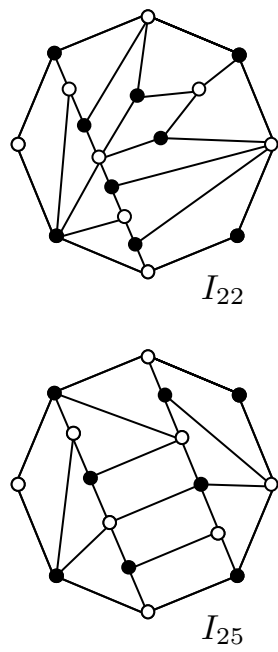
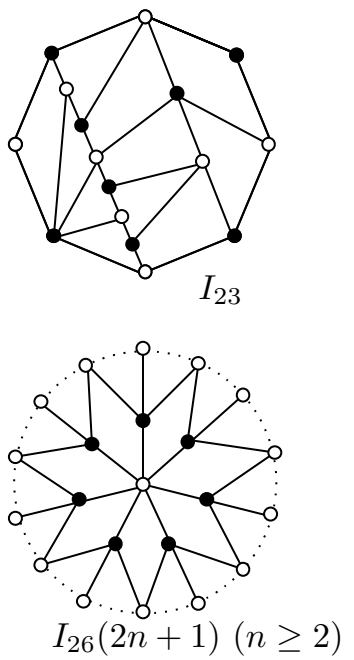

Figure 16: The 6 families of bipartite $\mathcal{P}$-irreducible quadrangulations without an attached cube. 


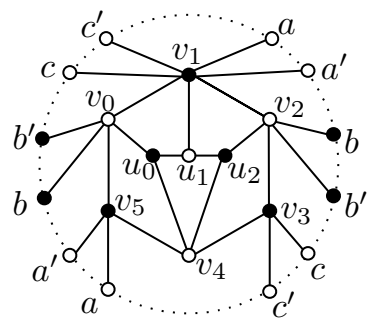

(i)

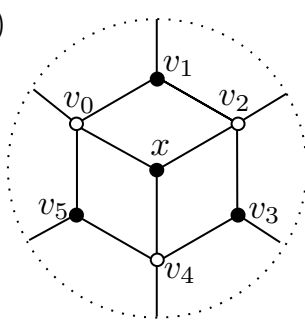

(iv)

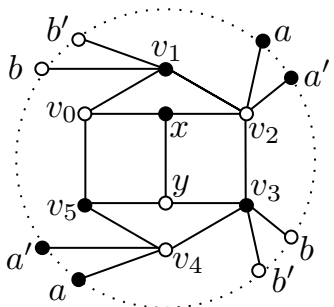

(ii)

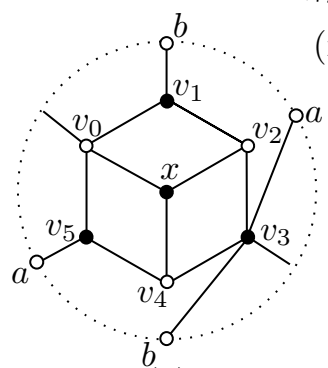

(v)

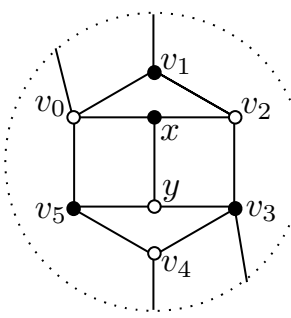

(iii)

Figure 17: Structures of bipartite $\mathcal{P}$-irreducible quadrangulations with no attached cube.

Case II. $G$ has two adjacent vertices $x$ and $y$ of degree 3: See the inside of the hexagon in (ii) of Figure 17. Note that each of $\operatorname{deg}\left(v_{0}\right), \operatorname{deg}\left(v_{2}\right), \operatorname{deg}\left(v_{3}\right)$ and $\operatorname{deg}\left(v_{5}\right)$ is at least 4 since there is no 2-path induced by vertices of degree 3 by the previous argument. As well as Case I, we consider face-contractions of $v_{1} v_{2} x v_{0}$ at $\left\{v_{1}, x\right\}$ and $v_{3} v_{4} v_{5} y$ at $\left\{v_{4}, y\right\}$. By Lemma 3.8, we have two diagonal 3-curves $\gamma$ and $\gamma^{\prime}$ passing through $\left\{v_{1}, x\right\}$ and $\left\{v_{4}, y\right\}$, respectively. We may assume that $\gamma$ passes $v_{3}$ as the third vertex, up to symmetry.

If $\gamma^{\prime}$ passes $v_{2}$, then it (resp., $\gamma$ ) goes through $v_{2} a v_{4} a^{\prime}$ (resp., $v_{1} b v_{3} b^{\prime}$ ) in (ii) of Figure 17. We consider the identification of vertices and further fill the blank non-quadrilateral regions, and obtain $I_{24}$ and $I_{25}$. On the other hand, if $\gamma^{\prime}$ passes $v_{0}$ as the third vertex, then both of $\gamma$ and $\gamma^{\prime}$ pass a common face $v_{0} v_{1} v_{4} v_{3}$ (see (iii) in the figure). However, we can fill the unique hexagonal region with neither $H_{1}, H_{2}$ nor $H_{3}$ in Figure 6.

Case III. All vertices of degree 3 are independent: Let $x$ be a vertex of degree 3 having neighbors $\left\{v_{0}, v_{2}, v_{4}\right\}$ and $v_{0} v_{1} v_{2} v_{3} v_{4} v_{5}$ as its link walk. By the assumption, each of $\operatorname{deg}\left(v_{0}\right), \operatorname{deg}\left(v_{2}\right)$ and $\operatorname{deg}\left(v_{4}\right)$ is at least 4 . We consider face-contractions of three faces incident to $x$ and have some cases depending on the forbidden structure of the resulting graph. (For example, if each operation yields multiple edges, we have (iv) in Figure 17, but it is immediately excluded since we can find a simple closed curve intersecting $G$ at only $\left\{v_{0}, v_{2}\right\}$.) Further, we try to identify vertices as well as the previous cases but most cases are not suited other than the following one case.

See (v) in Figure 17 that has the unique blank octagonal region $D$ bounded by a closed walk $v_{1} v_{2} v_{3} a v_{5} v_{4} v_{3} b$. Note that each of $\operatorname{deg}_{D}\left(v_{2}\right), \operatorname{deg}_{D}\left(v_{4}\right), \operatorname{deg}_{D}(a)$ and $\operatorname{deg}_{D}(b)$ is at least 1 , since $G$ has no vertex of degree 2 and no two adjacent vertices of degree 3 . Therefore, $D$ satisfies the conditions of Lemma 5.5. However, putting either $O_{9}(2 l+1)$ $(l \geq 1)$ or $O_{10}$ in Figure 13 into $D$ would yield two adjacent vertices of degree 3 . Actually, when filling $D$ with $O_{9}(2 l)(l \geq 1)$, we obtain $I_{26}(2 l+3)=I_{26}(2(l+1)+1)$. Then, we got the conclusion of the theorem. 
As well as $I_{18}\left[2 ; s_{1}, \ldots, s_{n}\right]$, we can naturally define $I_{18}\left[1 ; s_{1}, \ldots, s_{n}\right]$ by using (iii) of Figure 18 which also has the unique hexagonal region.

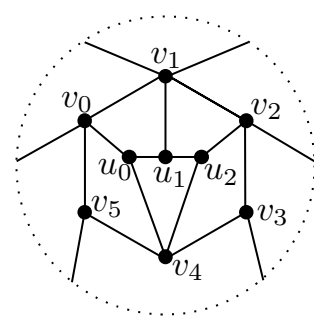

(i)

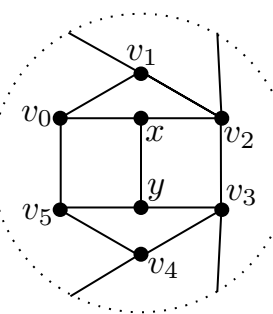

(ii)

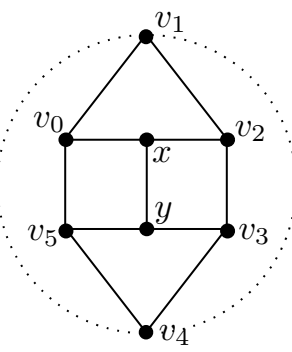

(iii)

Figure 18: Structures of nonbipartite $\mathcal{P}$-irreducible quadrangulations with no attached cube.

Theorem 7.4. Let $G$ be a nonbipartite $\mathcal{P}$-irreducible quadrangulation of the projective plane. If $G$ has no attached cube, then $G$ is isomorphic to $I_{18}[1 ; 1, \ldots, 1]$.

Proof. As well as the proof of Theorem 7.3, we divide our argument into the following three cases.

Case I. $G$ has a 2-path $u_{0} u_{1} u_{2}$ induced by three vertices of degree 3: See (i) in Figure 18. Since $G$ is nonbipartite, we have three semi-diagonal 3 -curves passing through $\left\{v_{0}, u_{1}, v_{2}\right\},\left\{v_{1}, u_{2}, u_{3}\right\}$ and $\left\{v_{1}, u_{0}, v_{5}\right\}$, respectively. (Consider the $2_{3}$-path shrink $u_{0} u_{1} u_{2}$ and face-contractions of $v_{2} v_{3} v_{4} u_{2}$ and $v_{4} v_{5} v_{0} u_{0}$.) Under the conditions, there should be three edges $v_{0} v_{2}, v_{1} v_{3}$ and $v_{1} v_{5}$ since $v_{0} v_{1} v_{2} v_{3} v_{4} v_{5}$ forms a cycle of $G$. By Lemma 4.3, the quadrilateral region $v_{1} v_{2} v_{0} v_{5}$ corresponds to a face of $G$. However, there is an essential simple closed curve passing through only $v_{0}$ and $v_{1}$, a contradiction.

Case II. $G$ has two adjacent vertices $x$ and $y$ of degree 3: See (ii) in Figure 18. Note that each of $\operatorname{deg}\left(v_{0}\right), \operatorname{deg}\left(v_{2}\right), \operatorname{deg}\left(v_{3}\right)$ and $\operatorname{deg}\left(v_{5}\right)$ is at least 4 . Suppose that $v_{0} v_{1} v_{2} v_{3} v_{4} v_{5}$ is a cycle of $G$. We consider the face-contraction of $v_{0} v_{1} v_{2} x$ (resp., $v_{3} v_{4} v_{5} y$ ) at $\left\{v_{1}, x\right\}$ (resp., $\left.\left\{v_{4}, y\right\}\right)$. Then, there are two semi-diagonal 3-curves and hence we have $v_{1} v_{3}, v_{2} v_{4} \in$ $E(G)$, up to symmetry. Clearly, we find an essential simple closed curve intersecting $G$ at only $\left\{v_{2}, v_{3}\right\}$, a contradiction.

Therefore, we assume that $v_{0} v_{1} v_{2} v_{3} v_{4} v_{5}$ is not a cycle of $G$. Under the conditions, $v_{1}$ and $v_{4}$ must coincide and the other vertices of the closed walk are distinct (see (iii) in Figure 18). Then the configuration contains a blank hexagonal region $v_{1} v_{2} v_{3} v_{4}\left(=v_{1}\right) v_{0} v_{5}$ and it satisfies the conditions of Lemma 5.3. Now, we apply the result of the lemma. But, $H_{19}$ is excluded immediately since it contains an attached cube. In this case, $H_{18}[1, \ldots, 1]$ only fits the region. The resulting graph is clearly $I_{18}[1 ; 1, \ldots, 1]$.

Case III. All vertices of degree 3 are independent: Do the same procedure as in the previous theorem. (Begin with considering face-contractions of three faces incident to a vertex of degree 3.) However, we obtain no $\mathcal{P}$-irreducible quadrangulation from this case; since two adjacent vertices of degree 3 often appear. Therefore, the theorem follows. 


\section{References}

[1] D. Archdeacon, J. Hutchinson, A. Nakamoto, S. Negam and K. Ota, Chromatic numbers of quadrangulations on closed surfaces, J. Graph Theory 37 (2001), 100-114, doi:10.1002/jgt. 1005.

[2] V. Batagelj, An inductive definition of the class of 3-connected quadrangulations of the plane, Discrete Math. 78 (1989), 45-53, doi:10.1016/0012-365x(89)90159-3.

[3] G. Brinkmann, S. Greenberg, C. Greenhill, B. D. McKay, R. Thomas and P. Wollan, Generation of simple quadrangulations of the sphere, Discrete Math. 305 (2005), 33-54, doi:10.1016/j. disc.2005.10.005.

[4] H. J. Broersma, A. J. W. Duijvestijn and F. Göbel, Generating all 3-connected 4-regular planar graphs from the octahedron graph, J. Graph Theory 17 (1993), 613-620, doi:10.1002/jgt. 3190170508.

[5] P. Eades, S.-H. Hong, N. Katoh, G. Liotta, P. Schweitzer and Y. Suzuki, A linear time algorithm for testing maximal 1-planarity of graphs with a rotation system, Theoret. Comput. Sci. $\mathbf{5 1 3}$ (2013), 65-76, doi:10.1016/j.tcs.2013.09.029.

[6] D. Hudák, T. Madaras and Y. Suzuki, On properties of maximal 1-planar graphs, Discuss. Math. Graph Theory 32 (2012), 737-747, doi:10.7151/dmgt.1639.

[7] J. P. Hutchinson, Three-coloring graphs embedded on surfaces with all faces even-sided, $J$. Comb. Theory Ser. B 65 (1995), 139-155, doi:10.1006/jctb.1995.1047.

[8] W. Liu and Y. Chen, Polyhedral embeddings of snarks with arbitrary nonorientable genera, Electron. J. Combin. 19 (2012), \#P14 (6 pages), https: / / www . combinatorics . org/ ojs/index.php/eljc/article/view/v19i3p14.

[9] B. Mohar, Existence of polyhedral embeddings of graphs, Combinatorica 21 (2001), 395-401, doi:10.1007/s004930100003.

[10] B. Mohar and N. Robertson, Flexibility of polyhedral embeddings of graphs in surfaces, $J$. Comb. Theory Ser. B 83 (2001), 38-57, doi:10.1006/jctb.2001.2036.

[11] B. Mohar and A. Vodopivec, On polyhedral embeddings of cubic graphs, Combin. Probab. Comput. 15 (2006), 877-893, doi:10.1017/s0963548306007607.

[12] T. Nagasawa, K. Noguchi and Y. Suzuki, No optimal 1-planar graph triangulates any nonorientable closed surface, J. Graph Theory 89 (2018), 350-360, doi:10.1002/jgt.22255.

[13] M. Nagashima, A. Nakamoto, S. Negami and Y. Suzuki, Generating 3-connected quadrangulations on surfaces, Ars Combin. 116 (2014), 371-384.

[14] A. Nakamoto, Irreducible quadrangulations of the Klein bottle, Yokohama Math. J. 43 (1995), 125-139, http://hdl. handle.net/10131/5655.

[15] A. Nakamoto, Irreducible quadrangulations of the torus, J. Comb. Theory Ser. B 67 (1996), 183-201, doi:10.1006/jctb.1996.0040.

[16] A. Nakamoto, Triangulations and Quadrangulations on Surfaces, Ph.D. thesis, Keio University, Japan, 1996.

[17] A. Nakamoto, Generating quadrangulations of surfaces with minimum degree at least $3, J$. Graph Theory 30 (1999), 223-234, doi:10.1002/(sici)1097-0118(199903)30:3〈223::aid-jgt7〉 3.3.co;2-d.

[18] A. Nakamoto and K. Ota, Note on irreducible triangulations of surfaces, J. Graph Theory 20 (1995), 227-233, doi:10.1002/jgt.3190200211. 
[19] A. Nakamoto and Y. Suzuki, Diagonal slides and rotations in quadrangulations on the sphere, Yokohama Math. J. 55 (2010), 105-112, http://hdl. handle. net/10131/ 00011358.

[20] S. Negami and A. Nakamoto, Diagonal transformations of graphs on closed surfaces, Sci. Rep. Yokohama Nat. Univ. Sect. I Math. Phys. Chem. 40 (1993), 71-97.

[21] K. Noguchi and Y. Suzuki, Relationship among triangulations, quadrangulations and optimal 1-planar graphs, Graphs Combin. 31 (2015), 1965-1972, doi:10.1007/s00373-015-1568-8.

[22] N. Robertson and R. Vitray, Representativity of surface embeddings, in: B. Korte, L. Lovász, H. J. Prömel and A. Schrijver (eds.), Paths, Flows, and VLSI-Layout, Springer, Berlin, volume 9 of Algorithms and Combinatorics, 1990 pp. 293-328, papers from the meeting held at the University of Bonn, Bonn, June 20 - July 1, 1988.

[23] Y. Suzuki, Optimal 1-planar graphs which triangulate other surfaces, Discrete Math. 310 (2010), 6-11, doi:10.1016/j.disc.2009.07.016.

[24] Y. Suzuki, Re-embeddings of maximum 1-planar graphs, SIAM J. Discrete Math. 24 (2010), 1527-1540, doi:10.1137/090746835.

[25] Y. Suzuki, Cube-contractions in 3-connected quadrangulations, Ars Math. Contemp. 10 (2016), 281-290, doi:10.26493/1855-3974.552.bf3.

[26] Y. Suzuki and T. Watanabe, Generating even triangulations of the projective plane, J. Graph Theory 56 (2007), 333-349, doi:10.1002/jgt.20269. 